\title{
The Newton Polygon and Elliptic Problems with Parameter
}

By Robert Denk and Reinhard Mennicken of Regensburg, and LeONid Volevich of Moscow

\begin{abstract}
In the study of the resolvent of a scalar elliptic operator, say, on a manifold without boundary there is a well-known Agmon-Agranovich-Vishik condition of ellipticity with parameter which guarantees the existence of a ray of minimal growth of the resolvent. The paper is devoted to the investigation of the same problem in the case of systems which are elliptic in the sense of Douglis-Nirenberg. We look for algebraic conditions on the symbol providing the existence of the resolvent set containing a ray on the complex plane. We approach the problem using the Newton polyhedron method. The idea of the method is to study simultaneously all the quasihomogeneous parts of the system obtained by assigning to the spectral parameter various weights, defined by the corresponding Newton polygon. On this way several equivalent necessary and sufficient conditions on the symbol of the system guaranteeing the existence and sharp estimates for the resolvent are found. One of the equivalent conditions can be formulated in the following form: all the upper left minors of the symbol satisfy ellipticity conditions. This subclass of systems elliptic in the sense of Douglis-Nirenberg was introduced by A. KozHevnikov [K2].
\end{abstract}

\section{Introduction}

We consider an operator on a manifold $M$ without boundary acting in the corresponding $L_{2}$-space and being defined by an elliptic matrix operator with smooth coefficients. In the study of spectral properties of this operator a natural question is the non-emptiness of the resolvent set, i. e., of the set $\Lambda \subset \mathbb{C}$ such that the operator $A(x, D)-\lambda I$ has a bounded inverse for $\lambda \in \Lambda$.

In the case of a scalar elliptic operator $A(x, D)$ the existence of a nontrivial resolvent set is provided by the so-called condition of ellipticity with parameter. This condition

1991 Mathematics Subject Classification. Primary: 47B25; Secondary: 47A11, 47A56

Keywords and phrases. Newton polygon, systems elliptic in the sense of Douglis-Nirenberg, systems elliptic with parameter. 
is due to Agmon and Agranovich - Vishik (see [Agm] and [AV]) and means that there exists a ray on the complex plane

$$
\mathcal{L}=\{\lambda \in \mathbb{C}: \lambda=\rho \exp (\mathrm{i} \vartheta), \rho>0\}
$$

such that

$$
A^{0}(x, \xi)-\lambda \neq 0 \quad\left((\lambda, \xi) \in \mathcal{L} \times \mathbb{R}^{n},|\xi|+|\lambda|>0, x \in M\right),
$$

where $A^{0}(x, \xi)$ denotes the principal symbol of the operator $A(x, D)$.

The condition above can be naturally extended to elliptic matrix operators of constant order $[\mathrm{AV}]$. In this case the principal symbol $A^{0}(x, \xi)$ is a matrix, whose elements are homogeneous polynomials in $\xi$ of constant order, and (1.2) must be replaced by

$$
\operatorname{det}\left(A^{0}(x, \xi)-\lambda I\right) \neq 0 \quad\left((\lambda, \xi) \in \mathcal{L} \times \mathbb{R}^{n},|\xi|+|\lambda|>0, x \in M\right) .
$$

Note that the left-hand side of (1.3) is a quasihomogeneous polynomial of $\xi$ and $\lambda$, and (1.3) means quasiellipticity of this polynomial.

It should be pointed out that Conditions (1.2), (1.3) permit us to use the calculus of pseudodifferential operators with parameter and to seek the resolvent as a series of such operators, where the norms of the remainders can be estimated by a constant times a negative power of $|\lambda|$ (the parametrix method). On this way it is possible not only prove that the equation

$$
A(x, D) u(x)-\lambda u(x)=f(x)
$$

on a compact manifold $M$ without boundary has a unique solution $u \in L_{2}$ for an arbitrary right - hand side $f \in L_{2}$, but also to obtain for this solution a two-sided a priori estimate in norms depending on the parameter $\lambda$.

Difficulties of principal character arise when one tries to extend the definition above to the case of matrix operators of variable order, i. e., to the systems elliptic in the sense of Douglis - Nirenberg. In this case the left - hand side of (1.3) is not a quasihomogeneous symbol and (1.3) must be replaced by a more adequate condition. ${ }^{1)}$

In his study of spectral asymptotics of systems elliptic in the sense of Douglis Nirenberg A. KozHevnikov (see [K1], [K2]) introduced an algebraic condition on the symbol (called parameter - ellipticity condition) which permitted him to prove the similarity of the system satisfying this condition to an almost diagonal system up to a symbol of order $-\infty$. The questions of solvability of (1.4) (i. e., the existence of the resolvent) and the validity of two-sided a priori estimates were not treated by him. In connection with this M. AGRANOVICH posed the problem of finding conditions on the symbol of a Douglis - Nirenberg elliptic system which make it possible to extend to these systems the above mentioned theory of elliptic systems of constant order with parameter (a priori estimates, solvability for large $|\lambda|$, estimates of the norms of remainders in parametrix series by negative powers of the parameter).

The present paper is devoted to the solution of this problem.

\footnotetext{
1) In the case of systems elliptic in the sense of Douglis-Nirenberg and having elements of the same order on the main diagonal the determinant (1.3) is also quasihomogeneous. This case was treated by Agranovich [A].
} 
For heuristic arguments, we consider a diagonal system

$$
A_{i i}(x, D) u_{i}-\lambda u_{i}=f_{i} \quad(i=1, \ldots, q)
$$

of elliptic operators $A_{i i}$ of various orders $r_{i}(i=1, \ldots, q)$. For simplicity here we consider this system in the whole space $\mathbb{R}^{n}$. We choose the indexing of equations (1.5) such that $r_{1} \geq r_{2} \geq \cdots \geq r_{q}$. It follows from [AV] that the existence of a ray (1.1) and a $\rho_{0}>0$ such that for $\lambda \in \mathcal{L},|\lambda|>\rho_{0}$, the equations (1.5) are uniquely solvable is equivalent to conditions (1.2) with $A^{0}(x, \xi)$ replaced by the principal symbols $A_{i i}^{0}(x, \xi), i=1, \ldots, q$. Because of the quasihomogeneity of $A_{i i}^{0}(x, \xi)-\lambda$ we can find positive constants $\delta_{i}, d_{i}$ such that for $\lambda \in \mathcal{L}$

$$
\delta_{i}\left(|\xi|^{r_{i}}+|\lambda|\right) \leq\left|A_{i i}^{0}(x, \xi)-\lambda\right| \leq d_{i}\left(|\xi|^{r_{i}}+|\lambda|\right) .
$$

Multiplying these estimates we obtain a two - sided estimate for $\operatorname{det}\left(A^{0}-\lambda I\right)$, where $A^{0}=\operatorname{diag}\left(A_{i i}^{0}\right)$, given by

$$
\delta \prod_{i=1}^{q}\left(|\xi|^{r_{i}}+|\lambda|\right) \leq\left|P^{0}(x, \xi, \lambda)\right| \leq d \prod_{i=1}^{q}\left(|\xi|^{r_{i}}+|\lambda|\right)
$$

here $\delta=\delta_{1} \cdot \ldots \cdot \delta_{q}, d=d_{1} \cdot \ldots \cdot d_{q}$, and

$$
P^{0}(x, \xi, \lambda)=\operatorname{det}\left(A^{0}(x, \xi)-\lambda I\right) .
$$

Denote by $N(P)$ the convex hull on the $(i, k)$-plane of the points

$$
(0,0),(0, q),\left(r_{1}, q-1\right),\left(r_{1}+r_{2}, q-2\right), \ldots,\left(r_{1}+\ldots+r_{q}, 0\right),
$$

and set

$$
\Xi_{N(P)}(\xi, \lambda)=\sum_{(i, k) \in N(P)}|\xi|^{i}|\lambda|^{k},
$$

where the summation is extended over all integral points of the polygon $N(P)$. The product $\prod_{i=1}^{q}\left(|\xi|^{r_{i}}+|\lambda|\right)$ can be estimated from above and from below by a constant times the function (1.8). In other words, (1.6) is equivalent to the estimate

$$
\delta_{0} \Xi_{N(P)}(\xi, \lambda) \leq\left|P^{0}(x, \xi, \lambda)\right| \leq d_{0} \Xi_{N(P)}(\xi, \lambda) .
$$

Therefore there exists a $\rho_{0}>0$ such that

$$
\delta_{1} \Xi_{N(P)}(\xi, \lambda) \leq|P(x, \xi, \lambda)| \leq d_{1} \Xi_{N(P)}(\xi, \lambda)
$$

holds for $\xi \in \mathbb{R}^{n}$ and $\lambda \in \mathcal{L}$ with $|\lambda| \geq \rho_{0}$, where

$$
P(x, \xi, \lambda)=\operatorname{det}(A(x, \xi)-\lambda I)=\sum b_{\alpha k}(x) \xi^{\alpha} \lambda^{k}
$$

In the following we associate with every polynomial symbol of variables $\xi$ and $\lambda$ the so-called Newton polygon $N(P)$ and the corresponding function (1.8). The symbols 
satisfying an estimate of the type (1.10) are called elliptic with parameter with respect to the Newton polygon or, more concisely, $N$-elliptic with parameter.

In the case of a nondiagonal matrix symbol $A(x, \xi)$ which is elliptic in the sense of Douglis - Nirenberg (here $r_{j}=l_{j}+m_{j}$, and the numbers $l_{j}, m_{j}, j=1, \ldots, q$, are defined by the Douglis - Nirenberg structure of the system, see Section 3), inequality (1.10) with symbol (1.11) can be taken as the definition of ellipticity with parameter.

The polynomials $P(\xi, \lambda)$ satisfying an estimate from below of the type (1.10) with the parameter $\lambda$ varying in various domains were investigated by MIKHAILOV and Gindikin - Volevich (see [GV] and the references therein). According to these results, necessary and sufficient conditions for the validity of the estimate (1.10) can be formulated in terms of the full set of quasihomogeneous parts of $P(\xi, \lambda)$.

For the given symbol on the right-hand side of (1.11) and $r>0$ we define the $r$ - degree $d_{r}(P)$ by

$$
d_{r}(P)=\max \left\{|\alpha|+r k: b_{\alpha k}(x) \not \equiv 0\right\} .
$$

Denote by $P_{r}(x, \xi, \lambda)$ the $r$-principal part of $P$ :

$$
P_{r}(x, \xi, \lambda)=\sum_{|\alpha|+r k=d_{r}(P)} b_{\alpha k}(x) \xi^{\alpha} \lambda^{k}
$$

This polynomial is $(1, r)$ - homogeneous in $(\xi, \lambda)$ of degree $d_{r}(P)$, i. e., we have

$$
P_{r}\left(x, t \xi, t^{r} \lambda\right)=t^{d_{r}(P)} P_{r}(x, \xi, \lambda) .
$$

A natural modification of the results stated in [GV] leads to the necessary and sufficient conditions for the estimate (1.10) to take place: for every $r>0$

$$
P_{r}(x, \xi, \lambda) \neq 0 \quad\left((\lambda, \xi) \in \mathcal{L} \times \mathbb{R}^{n},|\xi|>0,|\lambda|>0, x \in M\right) .
$$

Formally (1.12) contains an infinite number of conditions on the symbol. However (as it will be seen below), only a finite number of values of $r$ (defined by the Newton polygon) are essential.

Conditions (1.12) can be reformulated in terms of the matrix symbol $A(x, \xi)-\lambda I$. We assign to the variable $\lambda$ a "weight" $r$ and define the $r$-principal part $(A(x, \xi)-\lambda I)_{r}$ (in the sense of Leray-Douglis-Nirenberg) of the symbol $A(x, \xi)-\lambda I$. Then

$$
\operatorname{det}(A(x, \xi)-\lambda I)_{r}=P_{r}(x, \xi, \lambda) \text {. }
$$

Comparing (1.13) with (1.12) and with the explicit form of the symbol $(A(x, \xi)-\lambda I)_{r}$, we find that the condition of ellipticity with parameter formulated above is equivalent to the condition of KozHevNikov (see [K2]).

We show that the equivalent conditions of ellipticity with parameter also include sharp estimates for the elements of $(A(x, \xi)-\lambda I)^{-1}$. Combining these estimates with the standard calculus of pseudodifferential operators with parameter (see $[\mathrm{G}]$ and $[\mathrm{Sh}]$ ), we prove the unique solvability of (1.4) in the function spaces defined by the structure of the system. These spaces are endowed with norms which depend on the parameter $\lambda$. 
The plan of presentation is the following. In Section 2 all the needed facts about the Newton polygon of a polynomial are presented and $N$-ellipticity with parameter for polynomials is treated. Section 3 is devoted to the definition of ellipticity with parameter for polynomial matrices. We prove the equivalence of our and KozHEvNIKOV's definitions. In Section 4 the main theorem on the unique solvability of the system (1.4) is proved in $\mathbb{R}^{n}$ in the case of constant coefficients. The cases of variable coefficients in $\mathbb{R}^{n}$ and of a compact manifold without boundary are treated in Section 5 .

\section{The Newton polygon of a polynomial. $N$-ellipticity with parameter for polynomials}

\subsection{Definition of $N$-elliptic polynomials with parameter}

Consider a polynomial in the variables $\xi=\left(\xi_{1}, \ldots, \xi_{n}\right) \in \mathbb{R}^{n}$ and $\lambda \in \mathbb{C}$ given by

$$
P(\xi, \lambda)=\sum_{\alpha, k} a_{\alpha k} \xi^{\alpha} \lambda^{k}
$$

where $\alpha=\left(\alpha_{1}, \ldots, \alpha_{n}\right)$ and $\xi^{\alpha}=\xi_{1}^{\alpha_{1}} \cdot \ldots \cdot \xi_{n}^{\alpha_{n}}$. Denote by $\nu(P)$ the set of integral points $(i, k)$ such that $a_{\alpha k} \neq 0$, where $|\alpha|=\alpha_{1}+\cdots+\alpha_{n}=i$. Denote by $N(P)$ the convex hull of the union of $\nu(P) \cup\{(0,0)\}$ and the projections on the coordinate axes of the points belonging to $\nu(P)$. From the definition of $N(P)$ it follows that this polygon belongs to the positive quadrant of the plane, the origin is one of the vertexes of $N(P)$, and two sides of $N(P)$ lie on the coordinate lines. A detailed discussion of the Newton polygon can be found in [GV, Chapters 1 and 2].

Suppose that the polynomial (2.1) is solved with respect to the highest degree of $\lambda$, i. e.,

$$
P(\xi, \lambda)=\lambda^{q}+\sum_{k<q} \sum_{\alpha} a_{\alpha k} \xi^{\alpha} \lambda^{k} .
$$

The corresponding polygon $N(P)$ has no sides parallel to the $i$-axes and is of the form indicated in Fig. $1 \mathrm{a}$ ) and Fig. $1 \mathrm{~b})$.

As in the Introduction, we define the function

$$
\Xi_{P}(\xi, \lambda)=\sum_{(i, k) \in N(P)}|\xi|^{i}|\lambda|^{k} .
$$

Definition 2.1. The polynomial (2.2) is called $N$-elliptic with parameter if there exists a ray (1.1) and positive constants $d, \rho_{0}$ such that the estimate

$$
d \Xi_{P}(\xi, \lambda) \leq|P(\xi, \lambda)| \quad\left((\lambda, \xi) \in \mathcal{L} \times \mathbb{R}^{n},|\lambda| \geq \rho_{0}\right)
$$

holds.

The polynomials (2.2) satisfying (2.4) for $\lambda$ belonging to some lower half-plane $\left\{\lambda \in \mathbb{C}: \operatorname{Im} \lambda \leq \rho_{0}\right\}$ were studied in detail in [GV, Chapter 2]. They were called 


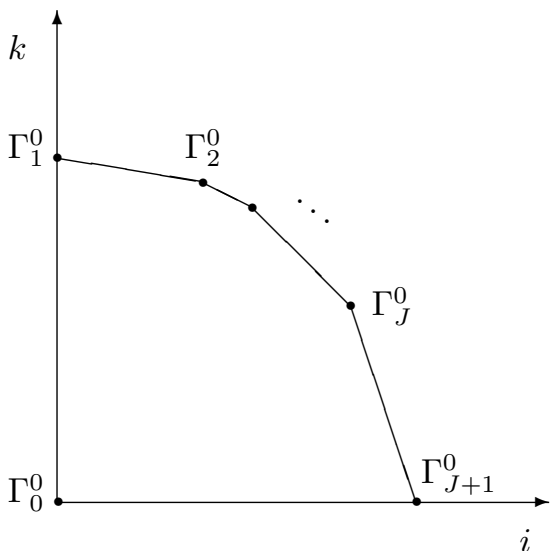

Fig. 1 a)

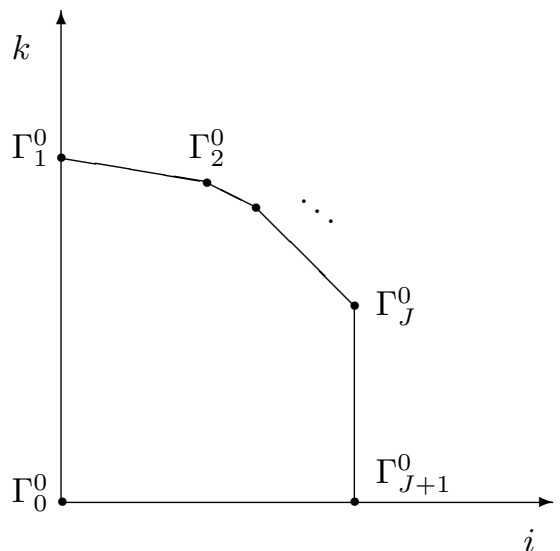

Fig. $1 \mathrm{~b}$ )

$N$-stable correct (if $N(P)$ is as indicated in Fig. 1 b)) and $N$-parabolic (if $N(P)$ is as indicated in Fig. $1 \mathrm{a})$ ).

We now introduce some simple notions connected with the Newton polygon.

\subsection{Leading and lower points of the Newton polygon}

Denote by $\Gamma_{j}^{0}=\left(i_{j}, k_{j}\right), j=0, \ldots, J+1$, the vertexes of $N(P)$ indexed in the clockwise direction beginning with the vertex $\Gamma_{0}^{0}=(0,0)$. Denote by $\Gamma_{j}^{1}, j=0, \ldots, J$, the side joining the vertexes $\Gamma_{j}^{0}$ and $\Gamma_{j+1}^{0}$ and by $\Gamma_{J+1}^{1}$ the side joining $\Gamma_{J+1}^{0}$ and $\Gamma_{0}^{0}$. The vertex $\Gamma_{1}^{0}$ belongs to the vertical axis and $\Gamma_{J+1}^{0}$ belongs to the horizontal one. We distinguish two cases, corresponding to Fig. $1 \mathrm{a}$ ) and $1 \mathrm{~b}$ ):

$$
\begin{aligned}
& i_{J+1}>i_{J}, \\
& i_{J+1}=i_{J} .
\end{aligned}
$$

In the first case the exterior normals to the sides of $N(P)$ not belonging to the coordinate axes have positive components. In the case (2.6) the side $\Gamma_{J}^{1}$ is vertical.

An integral point $(i, k) \in N(P)$ is called a lower point if there exists a point $\left(i^{\prime}, k^{\prime}\right) \in$ $N(P)$ (not necessarily integral) such that $i^{\prime} \geq i, k^{\prime} \geq k$ and one of the inequalities is strict. Otherwise, the integral point is called a leading point. Accordingly, the monomials $a_{\alpha k} \xi^{\alpha} \lambda^{k}$ in (2.1) are classified as leading or lower monomials.

The vertex $\Gamma_{j}^{0}$ is called leading if it is a leading point of $N(P)$. Accordingly, the side $\Gamma_{j}^{1}$ is called leading if it joins two leading vertexes. All the points belonging to a leading side are leading points of $N(P)$. Note that in the case (2.5) (cf. Fig. $1 \mathrm{a}$ )) all the vertexes $\Gamma_{1}^{0}, \ldots, \Gamma_{J+1}^{0}$ and the sides $\Gamma_{1}^{1}, \ldots, \Gamma_{J}^{1}$ joining them are leading. In the case (2.6) (cf. Fig $1 \mathrm{~b}$ )) the side $\Gamma_{J}^{1}$ and the vertex $\Gamma_{J+1}^{0}$ are not leading. 


\subsection{Principal parts of a polynomial connected with the Newton polygon}

Denote by $\nu^{(j)}, j=1, \ldots, J$, the vectors of the exterior normals to the sides $\Gamma_{j}^{1}, j=$ $1, \ldots, J$. Since the components of $\nu^{(j)}$ are defined up to a positive constant we can suppose that

$$
\nu^{(j)}=\left(1, r_{j}\right), \quad r_{j}=\left(i_{j+1}-i_{j}\right) /\left(k_{j}-k_{j+1}\right) .
$$

From obvious geometrical arguments it follows that

$$
r_{1}>\cdots>r_{J}>0 \quad \text { in the case }(2.5),
$$

$$
r_{1}>\cdots>r_{J-1}>r_{J}=0 \quad \text { in the case }(2.6) \text {. }
$$

For $j=2, \ldots, J$ denote by $V_{j}$ the open angle formed by the directions of $\nu^{(j-1)}$ and $\nu^{(j)}$. Then a vector $\nu=(1, r)$ belongs to $V_{j}$ if and only if $r_{j-1}>r>r_{j}$. The angle $V_{1}$ corresponding to the vertex $\Gamma_{1}^{0}$ is formed by the directions $(-1,0)$ and $\left(1, r_{1}\right)$.

With each vertex $\Gamma_{j}^{0}$ and each side $\Gamma_{j}^{1}$ we connect the corresponding principal part of the polynomial

$$
P_{\Gamma_{j}^{\kappa}}(\xi, \lambda)=\sum_{\substack{\alpha, k \\(|\alpha|, k) \in \Gamma_{j}^{\kappa}}} a_{\alpha k} \xi^{\alpha} \lambda^{k} \quad(\kappa=0,1) .
$$

In the Introduction we defined the $r$-degree $d_{r}(P)$ as $\max (|\alpha|+r k)$ extended over all monomials of $P$. We set

$$
P_{r}(\xi, \lambda)=\sum_{|\alpha|+r k=d_{r}(P)} a_{\alpha k} \xi^{\alpha} \lambda^{k} .
$$

It is easy to check that

$$
P_{r}(\xi, \lambda)=\lim _{\rho \rightarrow \infty} \rho^{-d_{r}(P)} P\left(\rho \xi, \rho^{r} \lambda\right) .
$$

There exists a close relation between the principal parts of $P$ in the sense of $(2.10)$ and (2.11). Namely, if the numbers $r_{j}$ are as in (2.7), then

$$
\begin{aligned}
& P_{\Gamma_{j}^{1}}(\xi, \lambda)=P_{r_{j}}(\xi, \lambda), \\
& P_{\Gamma_{j}^{0}}(\xi, \lambda)=P_{r}(\xi, \lambda) \quad\left((1, r) \in V_{j}\right) .
\end{aligned}
$$

\subsection{Main theorem}

Now we can formulate the main result of this section.

Theorem 2.2. For the polynomial (2.2) the following conditions are equivalent.

(I) The polynomial $P(\xi, \lambda)$ is $N$-elliptic with parameter, i. e., there exists a ray $\mathcal{L}$ and constants $d>0, \rho_{0}>0$ such that the estimate (2.4) holds.

(II) For arbitrary $r>0$,

$$
P_{r}(\xi, \lambda) \neq 0 \quad\left((\lambda, \xi) \in \mathcal{L} \times \mathbb{R}^{n},|\xi|>0,|\lambda|>0\right) .
$$


(III) For each leading vertex $\Gamma_{j}^{0}$ and each leading side $\Gamma_{j}^{1}$

$$
P_{\Gamma_{j}^{\kappa}}(\xi, \lambda) \neq 0 \quad\left((\lambda, \xi) \in \mathcal{L} \times \mathbb{R}^{n},|\xi|>0,|\lambda|>0, \kappa=0,1\right) .
$$

Remark 2.3. The connection between estimates from below of polynomials by means of their monomials and "quasiellipticity" of all principal parts was found by Mikhailov. A number of results of such type can be found in [GV]. The above theorem is a generalization of [GV, Theorem 2.4.2]. The proof we present is based on the geometrical construction used in [GV, Chapter 4].

Proof of Theorem 2.2. (I) $\Rightarrow$ (II). For $j=1, \ldots, J$ and $\kappa=0,1$ we set

$$
\Xi_{\Gamma_{j}^{\kappa}}(\xi, \lambda)=\sum_{(i, k) \in \Gamma_{j}^{\kappa}}|\xi|^{i}|\lambda|^{k} .
$$

From the definition of the $r$-degree $d_{r}(P)$ and (2.13), (2.14) it follows that

$$
P\left(\rho \xi, \rho^{r} \lambda\right)=\rho^{d_{r}(P)} P_{\Gamma_{j}^{\kappa}}(\xi, \lambda)+o\left(\rho^{d_{r}(P)}\right)
$$

and

$$
\Xi_{N(P)}\left(\rho \xi, \rho^{r} \lambda\right)=\rho^{d_{r}(P)} \Xi_{\Gamma_{j}^{\kappa}}(\xi, \lambda)+o\left(\rho^{d_{r}(P)}\right)
$$

hold for $r=r_{j}$ if $\kappa=1$ and for $(1, r) \in V_{j}$ if $\kappa=0$.

To prove (2.15), for $r>0$ and $\rho \geq 1$ we replace $(\xi, \lambda)$ in $(2.4)$ by $\left(\rho \xi, \rho^{r} \lambda\right)$. If we divide both sides of the resulting estimate by $\rho^{d_{r}(P)}$ and use $(2.17),(2.18)$, then for $\rho \rightarrow \infty$ we come to $(2.15)$.

(II) $\Rightarrow$ (III) trivially follows from (2.13) and (2.14).

$(\mathrm{III}) \Rightarrow(\mathrm{I})$. We fix $\varepsilon>0$. For each leading vertex $\Gamma_{j}^{0}=\left(i_{j}, k_{j}\right)$ we choose an open subdomain $U_{j}^{0}$ of the $(|\xi|,|\lambda|)$-plane with the following property: for each integral point $(i, k) \in N(P) \backslash \Gamma_{j}^{0}$ and for each $(|\xi|,|\lambda|) \in U_{j}^{0}$ we have

$$
|\xi|^{i}|\lambda|^{k} \leq \varepsilon|\xi|^{i_{j}}|\lambda|^{k_{j}}
$$

Similarly, for each segment $\Gamma_{j}^{1}$ we choose an open subdomain $U_{j}^{1}$ such that for each integral point $(i, k) \in N(P) \backslash \Gamma_{j}^{1}$ and for each $(|\xi|,|\lambda|) \in U_{j}^{1}$ we have

$$
|\xi|^{i}|\lambda|^{k} \leq \varepsilon \sum_{(p, q) \in \Gamma_{j}^{1}}|\xi|^{p}|\lambda|^{q}
$$

where the sum on the right-hand side is extended over all integral points of the segment $\Gamma_{j}^{1}$. Now we use the fact that for each $\varepsilon>0$ the subdomains $U_{j}^{0}$ and $U_{j}^{1}$ can be chosen in a way such that for some $\rho_{0}=\rho_{0}(\varepsilon)$ we have

$$
\left\{(|\xi|,|\lambda|) \in \mathbb{R}_{+}^{2}:|\lambda| \geq \rho_{0}\right\} \subset \bigcup_{j=1}^{J} U_{j}^{0} \cup \bigcup_{j=1}^{J-1} U_{j}^{1} .
$$


The proof of this result can be found in [GV, Subsections 4.2.3-4.2.5]. Due to this fact, it is sufficient to prove the estimate $(2.4)$ separately for $(\xi, \lambda) \in \widehat{U}_{j}^{\kappa}(\kappa=0,1)$, where we have set

$$
\widehat{U}_{j}^{\kappa}=\left\{(\xi, \lambda) \in \mathbb{R}^{n} \times \mathcal{L}:(|\xi|,|\lambda|) \in U_{j}^{\kappa}\right\} \quad(\kappa=0,1) .
$$

Estimate in $\widehat{U}_{j}^{0}$ : According to the definition (2.10),

$$
P_{\Gamma_{j}^{0}}(\xi, \lambda)=\sum_{|\alpha|=i_{j}} a_{\alpha, k_{j}} \xi^{\alpha} \lambda^{k_{j}}=\pi_{j}^{0}(\xi) \lambda^{k_{j}},
$$

where $\pi_{j}^{0}(\xi)$ is a homogeneous polynomial of degree $i_{j}$. According to (2.16) this polynomial is elliptic and, consequently, there exists a $\delta_{j}^{0}>0$ such that

$$
\left|P_{\Gamma_{j}^{0}}(\xi, \lambda)\right| \geq \delta_{j}^{0}|\xi|^{i_{j}}|\lambda|^{k_{j}}
$$

Taking into account (2.19), we obtain

$$
\begin{aligned}
|P(\xi, \lambda)| & \geq\left|P_{\Gamma_{j}^{0}}(\xi, \lambda)\right|-\left|P(\xi, \lambda)-P_{\Gamma_{j}^{0}}(\xi, \lambda)\right| \\
& \geq \delta_{j}^{0}|\xi|^{i_{j}}|\lambda|^{k_{j}}-K \sum_{(i, k) \in N(P) \backslash \Gamma_{j}^{0}}|\xi|^{i}|\lambda|^{k} \\
& \geq\left[\delta_{j}^{0}-K \varepsilon(\kappa(P)-1)\right]|\xi|^{i_{j}}|\lambda|^{k_{j}} \\
& \geq \frac{1}{2} \delta_{j}^{0}|\xi|^{i_{j}}|\lambda|^{k_{j}}, \quad(\xi, \lambda) \in \widehat{U}_{j}^{0},
\end{aligned}
$$

if $\varepsilon$ is small enough (here $\kappa(P)$ is the number of integral points in $N(P)$ and the constant $K$ depends only on the polynomial (2.1)).

On the other side, it follows from (2.19) that for $(\xi, \lambda) \in \widehat{U}_{j}^{0}$

$$
\sum_{(i, k) \in N(P)}|\xi|^{i}|\lambda|^{k} \leq(1+(\kappa(P)-1) \varepsilon)|\xi|^{i_{j}}|\lambda|^{k_{j}} .
$$

Comparing the above estimates we obtain $(2.4)$ in $\widehat{U}_{j}^{0}$.

Estimate in $\widehat{U}_{j}^{1}$ : According to $(2.13)$, the polynomial $P_{\Gamma_{j}^{1}}(\xi, \lambda)$ can be written in the form

$$
\begin{aligned}
P_{\Gamma_{j}^{1}}(\xi, \lambda) & =\sum_{|\alpha|+r_{j} k=d_{r_{j}}(P)} a_{\alpha k} \xi^{\alpha} \lambda^{k} \\
& =\pi_{j}^{0}(\xi) \lambda^{k_{j}}+\cdots+\pi_{j+1}^{0}(\xi) \lambda^{k_{j+1}} \\
& =\pi_{j}^{0}(\xi) \lambda^{k_{j+1}} \pi_{j}^{1}(\xi, \lambda),
\end{aligned}
$$

where $\pi_{j}^{1}(\xi, \lambda)$ is a $\left(1, r_{j}\right)$-homogeneous function of degree $i_{j+1}-i_{j}$. It follows from (2.16) and the $\left(1, r_{j}\right)$-homogeneity that $\pi_{j}^{1}(\xi, \lambda)$ can be estimated from below by const $\left(|\xi|^{i_{j+1}-i_{j}}+|\lambda|^{k_{j}-k_{j+1}}\right)$. Hence

$$
\left|P_{\Gamma_{j}^{1}}(\xi, \lambda)\right| \geq \delta_{j}^{1} \sum_{(i, k) \in \Gamma_{j}^{1}}|\xi|^{i}|\lambda|^{k} .
$$


Using this inequality we can literally repeat the argument in the proof of (2.4) for $\widehat{U}_{j}^{0}$ and establish the desired inequality in $\widehat{U}_{j}^{1}$. Thus the theorem is proved.

\section{Polynomial matrices elliptic with parameter}

\subsection{Polynomial matrices elliptic in the sense of Douglis - Nirenberg}

Consider a $q \times q$ matrix

$$
A(\xi)=\left(A_{i j}(\xi)\right)_{i, j=1, \ldots, q},
$$

whose elements $A_{i j}$ are polynomials of degree $\alpha_{i j}$ :

$$
\alpha_{i j}=\operatorname{deg} A_{i j}(\xi) \quad(i, j=1, \ldots, q) .
$$

As the degree of a product of two polynomials is the sum of their degrees, it is natural to set $\alpha_{i j}=-\infty$ in the case $A_{i j}(\xi) \equiv 0$.

For a given permutation $\pi$ of the numbers $1, \ldots, q$ we define

$$
R(\pi)=\alpha_{1 \pi(1)}+\cdots+\alpha_{q \pi(q)}
$$

and consider the maximum of these numbers over all permutations:

$$
R=\max _{\pi} R(\pi)
$$

Proposition 3.1. [V1, V2]. Let $\left\{\alpha_{i j}: i, j=1, \ldots, q\right\}$ be a given set of integers and $R$ be defined by (3.3). Then there exists a system of integers $m_{1}, \ldots, m_{q}, l_{1}, \ldots, l_{q}$ such that

$$
\begin{aligned}
& \alpha_{i j} \leq l_{i}+m_{j} \quad(i, j=1, \ldots, q), \\
& \sum_{i=1}^{q}\left(l_{i}+m_{i}\right)=R .
\end{aligned}
$$

The numbers $m_{1}, \ldots, l_{q}$ defined above permit us to define the Leray - Douglis - Nirenberg principal part of the polynomial matrix (3.1).

Denote by $A_{i j}^{0}(\xi)$ the homogeneous part of $A_{i j}(\xi)$ of order $l_{i}+m_{j}$, and set $A_{i j}^{0}(\xi) \equiv 0$ in the case $\alpha_{i j}<l_{i}+m_{j}$. The matrix

$$
A^{0}(\xi)=\left(A_{i j}^{0}(\xi)\right)_{i, j=1, \ldots, q}
$$

is called the principal part of $(3.1)$.

Definition 3.2. [V1, V2]. The polynomial matrix (3.1) is called nondegenerate if

$$
R=\operatorname{deg} \operatorname{det} A(\xi) \text {. }
$$


This definition means that in the calculation of

$$
\operatorname{det} A(\xi)=\sum_{\pi} \pm A_{1 \pi(1)}(\xi) \ldots A_{q \pi(q)}(\xi)
$$

the leading terms do not cancel. Note that in the case $R>\operatorname{deg} \operatorname{det} A(\xi)$ the above defined principal part is of no use.

It follows from (3.4), (3.5) that for a nondegenerate matrix (3.1)

$$
\operatorname{det} A^{0}(\xi)=\text { principal part of } \operatorname{det} A(\xi) .
$$

Definition 3.3. The matrix (3.1) is called elliptic in the sense of Douglis - Nirenberg if the following conditions are satisfied.

(I) $A(\xi)$ is a nondegenerate matrix.

(II) $P(\xi)=\operatorname{det} A(\xi)$ is an elliptic polynomial, i. e.,

$$
P_{0}(\xi) \neq 0 \text { for } \xi \in \mathbb{R}^{n} \backslash\{0\},
$$

where $P_{0}(\xi)$ is the principal part of $P(\xi)$.

The numbers $m_{1}, \ldots, l_{q}$ in Proposition 3.1 are defined up to the transformation

$$
\left\{m_{1}, \ldots, m_{q}, l_{1}, \ldots, l_{q}\right\} \longmapsto\left\{m_{1}+\kappa, \ldots, m_{q}+\kappa, l_{1}-\kappa, \ldots, l_{q}-\kappa\right\}
$$

so without loss of generality we can suppose that the numbers in one group, say, $m_{1}, \ldots, m_{q}$ are nonnegative. In general, the other numbers $l_{1}, \ldots, l_{q}$ can be either positive or negative. If some of the numbers $l_{1}, \ldots, l_{q}$ are negative, we have difficulties to consider the so-called $L_{2}$-realization of the corresponding matrix differential operator with the domain (see $[\mathrm{GG}]$ ):

$$
D_{A}=\left\{u \in L_{2}: A(D) u \in L_{2}\right\} .
$$

To obtain a definition of an elliptic matrix with nonnegative numbers in (3.4), (3.5) we define

$$
\alpha_{i j}^{\prime}= \begin{cases}\alpha_{i j}=\operatorname{deg} A_{i j} & \text { if } \quad A_{i j} \neq 0, \\ 0 & \text { if } \quad A_{i j} \equiv 0,\end{cases}
$$

and

$$
R^{\prime}=\max _{\pi}\left(\alpha_{1 \pi(1)}^{\prime}+\cdots+\alpha_{q \pi(q)}^{\prime}\right) .
$$

According to Proposition 3.1, we can define integers $m_{1}, \ldots, l_{q}$ satisfying the relations

$$
\begin{aligned}
& \alpha_{i j}^{\prime} \leq l_{i}+m_{j} \quad(i, j=1, \ldots, q) \\
& \sum_{i=1}^{q}\left(l_{i}+m_{i}\right)=R^{\prime}
\end{aligned}
$$

We can choose the constant $\kappa$ in (3.9) such that

$$
\min l_{i}=l_{i_{0}}=0 .
$$


Then inequalities (3.12) with $i=i_{0}$ show that $m_{j} \geq \alpha_{i_{0} j}^{\prime} \geq 0$ for $j=1, \ldots, q$.

Definition 3.4. The matrix (3.1) is called positively nondegenerate if

$$
R^{\prime}=\operatorname{deg} \operatorname{det} A(\xi) .
$$

If the condition of Definition 3.4 is fulfilled, we can suppose that all numbers $m_{1}, \ldots, l_{q}$ are nonnegative. Moreover, by changing the indexing of the rows and the columns of the matrix (3.1) we can suppose that

$$
m_{1} \geq m_{2} \geq \cdots \geq m_{q} \geq 0, \quad l_{1} \geq l_{2} \geq \cdots \geq l_{q} \geq 0,
$$

and therefore

$$
r_{1} \geq r_{2} \geq \cdots \geq r_{q} \geq 0, \quad r_{j}=l_{j}+m_{j}, \quad(j=1, \ldots, q) .
$$

In the following, we assume without additional stipulation that (3.14) and, consequently, (3.15) hold.

\subsection{Conditions of ellipticity with parameter for polynomial matrices}

Now we consider matrices of the form

$$
A(\xi)-\lambda I
$$

where $A(\xi)$ satisfies the conditions of the preceding subsection. As the definition of ellipticity, the definition of the ellipticity with parameter will contain a condition on the determinant of (3.16) and an analogue of the nondegeneracy condition of Definition 3.3 .

Consider the determinant of (3.16),

$$
P(\xi, \lambda)=\operatorname{det}(A(\xi)-\lambda I)=(-\lambda)^{q}+\sum_{j=1}^{q} P_{j}(\xi)(-\lambda)^{q-j}
$$

From the rule of calculation of the determinant and (3.15) it follows that

$$
\operatorname{deg} P_{j}(\xi) \leq r_{1}+\cdots+r_{j} \quad(j=1, \ldots, q) .
$$

Definition 3.5. The matrix (3.16) is called elliptic with parameter if the conditions below are satisfied:

(i) the Newton polygon $N(P)$ of the polynomial (3.17) contains the points

$$
(0,0),(0, q),\left(r_{1}, q-1\right),\left(r_{1}+r_{2}, q-2\right), \ldots,\left(r_{1}+\cdots+r_{q}, 0\right) ;
$$

(ii) the determinant (3.17) is $N$-elliptic with parameter (cf. Definition 2.1), i. e., there exists a ray (1.1) and positive constants $d, \rho_{0}$ such that

$$
d \Xi_{P}(\xi, \lambda) \leq|P(\xi, \lambda)| \text { for }(\lambda, \xi) \in \mathcal{L} \times \mathbb{R}^{n},|\lambda| \geq \rho_{0} .
$$


Remark 3.6. According to (3.18), the polygon $N(P)$ is contained in the convex hull of the points (3.19). Hence, condition (i) of the definition above can be reformulated as

(i') $N(P)$ coincides with the convex hull of the points (3.19).

If the conditions of Definition 3.5 are satisfied, then the estimate

$$
d_{1} \prod_{j=1}^{q}\left(|\xi|^{r_{j}}+|\lambda|\right) \leq|P(\xi, \lambda)| \quad\left((\lambda, \xi) \in \mathcal{L} \times \mathbb{R}^{n},|\lambda| \geq \rho_{0}\right)
$$

holds for a positive constant $d_{1}$. Indeed, removing the parenthesis we can rewrite the left - hand side of (3.21) in the form

$$
d_{1}\left(|\lambda|^{q}+\sum_{j=1}^{q} \sum_{\substack{s_{1}, \cdots, s_{j}=1 \\ s_{i} \neq s_{k}}}^{q}|\xi|^{r_{s_{1}}+\ldots+r_{s_{j}}}|\lambda|^{q-j}\right) .
$$

Note that in view of (3.15) we have $r_{s_{1}}+\cdots+r_{s_{j}} \leq r_{1}+\cdots+r_{j}$, and the points $\left(r_{s_{1}}+\cdots+r_{s_{j}}, q-j\right)$ belong to $N(P)$. Hence the terms $|\xi|^{r_{s_{1}}+\cdots+r_{s_{j}}}|\lambda|^{q-j}$ are contained in the sum $\Xi_{P}(\xi, \lambda)$ (see $\left.(2.3)\right)$, and (3.21) follows from (3.20).

Proposition 3.7. Conditions (i), (ii) of Definition 3.5 hold if and only if there exist a ray (1.1) and positive $d_{1}, \rho_{0}$ such that (3.21) is valid.

We have already proved the necessity. The proof of the sufficiency is based on two elementary lemmas.

Lemma 3.8. (cf. [GV, Lemma 1.2.1]). Let the point $(a, b)$ belong to the convex hull of the nonnegative points $\left(a_{j}, b_{j}\right), j=1, \ldots, J$. Then

$$
|\xi|^{a}|\lambda|^{b} \leq \sum_{j=1}^{J}|\xi|^{a_{j}}|\lambda|^{b_{j}}
$$

Proof. According to the definition of the convex hull of a finite set, there exist nonnegative numbers $\kappa_{1}, \ldots, \kappa_{J}, \kappa_{1}+\cdots+\kappa_{J}=1$, such that $a=\sum \kappa_{j} a_{j}$ and $b=$ $\sum \kappa_{j} b_{j}$. Then, cf. [H, (2.5.2)],

$$
|\xi|^{a}|\lambda|^{b}=\prod_{j=1}^{J}\left(|\xi|^{a_{j}}|\lambda|^{b_{j}}\right)^{\kappa_{j}} \leq \sum_{j=1}^{J} \kappa_{j}|\xi|^{a_{j}}|\lambda|^{b_{j}} .
$$

Lemma 3.9. (cf. [GV, Lemma 1.2.2]). Let $P(\xi, \lambda)$ be a polynomial of the form $(2.2)$, and assume that for some $\left(i_{0}, k_{0}\right), i_{0} \geq 0, k_{0} \geq 0, d$, and $\rho_{0}$ the inequality

$$
d|\xi|^{i_{0}}|\lambda|^{k_{0}} \leq|P(\xi, \lambda)| \quad\left((\lambda, \xi) \in \mathcal{L} \times \mathbb{R}^{n},|\lambda| \geq \rho_{0}\right)
$$

is valid. Then $\left(i_{0}, k_{0}\right) \in N(P)$. 
Proof. If $\left(i_{0}, k_{0}\right)$ does not belong to the polygon $N(P)$, then there exists a vector $\left(q_{1}, q_{2}\right)$ with $q_{1}, q_{2} \geq 0$ such that for some $\kappa>0$ we have

$$
q_{1} i+q_{2} k \leq \kappa \text { for }(i, k) \in N(P) \text { and } q_{1} i_{0}+q_{2} k_{0}>\kappa .
$$

Replacing $\xi$ and $\lambda$ in $(3.22)$ by $\xi t^{q_{1}}, \lambda t^{q_{2}}$ and passing to the limit as $t \rightarrow+\infty$, we arrive at a contradiction to $(3.22)$.

Pro of of Proposition 3.7. Sufficiency. Suppose that (3.21) holds. According to Lemma 3.9 all the points (3.19) belong to $N(P)$, and hence (see Remark 3.6) $N(P)$ coincides with the convex hull of (3.19). Using Lemma 3.8 we estimate the left - hand side of $(3.20)$ by a constant times

$$
|\lambda|^{q}+\sum_{j=1}^{q}|\xi|^{r_{1}+\cdots+r_{j}}|\lambda|^{q-j}
$$

and, consequently, by the left - hand side of (3.21).

If we set

$$
G(\xi, \lambda)=(A(\xi)-\lambda I)^{-1}, \quad G(\xi, \lambda)=\left(G_{i j}(\xi, \lambda)\right)_{i, j=1, \ldots, q},
$$

then Definition 3.5 can also be reformulated using inequalities for the elements of the matrix (3.23).

Proposition 3.10. For a matrix (3.1) satisfying (3.2), (3.4), (3.5) and (3.14) the conditions of Definition 3.5 hold if and only if for $\lambda \in \mathcal{L}$ with sufficiently large $|\lambda|$ the estimates

$$
\begin{aligned}
\left|G_{i j}(\xi, \lambda)\right| & \leq \operatorname{const}(1+|\xi|)^{l_{i}+m_{j}}\left(|\xi|^{r_{i}}+|\lambda|\right)^{-1}\left(|\xi|^{r_{j}}+|\lambda|\right)^{-1} \quad(i \neq j) \\
\left|G_{i i}(\xi, \lambda)\right| & \leq \mathrm{const}\left(|\xi|^{r_{i}}+|\lambda|\right)^{-1}
\end{aligned}
$$

hold.

Proof. Necessity. According to the definition of the inverse matrix,

$$
G_{i j}(\xi, \lambda)=P^{-1}(\xi, \lambda) \operatorname{det}(A(\xi)-\lambda I)^{(j, i)},
$$

where the $(q-1) \times(q-1)$ matrix $(A(\xi)-\lambda I)^{(j, i)}$ is obtained by canceling the $j$ - th row and the $i$-th column in (3.16). The determinant of this matrix is a polynomial in $\xi$ of degree not greater than

$$
l_{i}+m_{j}+\sum_{s \neq i, j} r_{s}
$$

and a polynomial in $\lambda$ of degree not greater than $q-2$ (if $i \neq j)$ and $q-1$ (if $i=j$ ). Replacing $|\xi|^{r_{i}}$ by $|\xi|^{r_{i}}+|\lambda|$ we estimate the determinant of $(A-\lambda I)^{(j, i)}$ by

$$
\text { const }(1+|\xi|)^{l_{i}+m_{j}} \prod_{s \neq i, j}\left(|\xi|^{r_{s}}+|\lambda|\right)
$$


for $i \neq j$ and by

$$
\text { const } \prod_{s \neq i}\left(|\xi|^{r_{s}}+|\lambda|\right)
$$

for $i=j$.

Using the estimate (3.21) we obtain (3.24), (3.25).

Sufficiency. The estimates $(3.24)$, (3.25) permit us to estimate from above the determinant of $G(\xi, \lambda)$ by

$$
\text { const } \prod_{j=1}^{q}\left(|\xi|^{r_{j}}+|\lambda|\right)^{-1} .
$$

Since $\operatorname{det} G(\xi, \lambda)=P^{-1}(\xi, \lambda)$, we obtain the estimate (3.21). Proposition 3.7 proves our statement.

Let us note that in the case $r_{i}>0, r_{j}>0$ the above estimates can be replaced by the stronger inequality

$$
\left|G_{i j}(\xi, \lambda)\right| \leq \operatorname{const}\left(|\xi|^{r_{i}}+|\lambda|\right)^{-\frac{m_{i}}{r_{i}}}\left(|\xi|^{r_{j}}+|\lambda|\right)^{-\frac{l_{j}}{r_{j}}} .
$$

This estimate will have sense also in the case when either $r_{i}=0$ or $r_{j}=0$ if we formally set $\frac{m_{i}}{r_{i}}=1$ or $\frac{l_{j}}{r_{j}}=1$, respectively. In the case $r_{i}=r_{j}=0$ the estimates (3.24), (3.25) should be replaced by

$$
\left|G_{i j}(\xi, \lambda)\right| \leq \text { const }|\lambda|^{-1}
$$

\subsection{The Kozehvnikov conditions}

According to Theorem 2.2, the estimate (3.20) holds if and only if conditions (2.16) are satisfied. Now we reformulate these conditions in terms of determinants of "principal parts" of $A-\lambda I$. On this way we come to the KozHEVNIKOV conditions [K2]. For simplicity we begin our analysis with the case of strict inequalities in (3.15), i. e.,

$$
r_{1}>r_{2}>\cdots>r_{q} \geq 0 \text {. }
$$

If (3.28) holds, all the points (3.19) are vertexes of $N(P)$, and the segments that connect them are the sides.

If, in addition, $r_{q}>0$, then all the points (3.19) (except the origin) are leading vertexes, and the conditions of Theorem 2.2 can be written in the form

$$
\begin{aligned}
P_{r_{\kappa}}(\xi, \lambda) \neq 0 \quad\left((\lambda, \xi) \in \mathcal{L} \times \mathbb{R}^{n},|\lambda|>0,|\xi|>0, \kappa=1, \ldots, q\right) \\
P_{r}(\xi, \lambda) \neq 0 \quad\left((\lambda, \xi) \in \mathcal{L} \times \mathbb{R}^{n},|\lambda|>0,|\xi|>0\right)
\end{aligned}
$$

where in (3.30) we consecutively take the values of $r$ satisfying the following inequalities

$$
r_{j}>r>r_{j+1} \quad(j=0, \ldots, q) \text {; }
$$

here we formally set $r_{0}=\infty$ and $r_{q+1}=0$. 
Following KozHevnikov, for given $\kappa=1, \ldots, q$ we denote by $A(\kappa)(\xi)$ the block

$$
A(\kappa)(\xi)=\left(A_{i j}(\xi)\right)_{i, j=1, \ldots, \kappa},
$$

and denote by $A^{0}(\kappa)(\xi)$ the corresponding principal part (defined by means of the numbers $\left.l_{1}, \ldots, l_{\kappa}, m_{1}, \ldots, m_{\kappa}\right)$

$$
A^{0}(\kappa)(\xi)=\left(A_{i j}^{0}(\xi)\right)_{i, j=1, \ldots, \kappa} .
$$

Lemma 3.11. Let (3.28) and (3.31) be satisfied. Then the relation

$$
P_{r}(\xi, \lambda)=(-\lambda)^{q-\kappa} \operatorname{det} A^{0}(\kappa)(\xi) \quad\left(r_{\kappa}>r>r_{\kappa+1}\right)
$$

holds for $\kappa=1, \ldots, q$. In the case $r_{q}=0$, relation (3.34) is true for $\kappa \leq q-1$.

Proof. According to the definition of the $r$-principal part we calculate $P_{r}$ attaining the weight $r$ to the variable $\lambda$ and take in (2.2) the monomials which $(1, r)$-order equal to $d_{r}(P)=r_{1}+\cdots+r_{\kappa}+(q-\kappa) r$.

Now we want to construct a matrix whose determinant equals to the left - hand side of (3.34). We set

$$
\alpha_{i j}(\kappa)= \begin{cases}\alpha_{i j} & \text { if } i \neq j \text { or } i=j=1, \ldots, \kappa, \\ r & \text { if } i=j=\kappa+1, \ldots, q .\end{cases}
$$

To obtain the principal part of $A(\xi)-\lambda I$ corresponding to the degrees $\alpha_{i j}(\kappa)$, we consider the system of relations

$$
\begin{aligned}
& l_{i}(\kappa)+m_{j}(\kappa) \geq \alpha_{i j}(\kappa) \quad(i, j=1, \ldots, q), \\
& \sum_{i=1}^{q}\left(l_{i}(\kappa)+m_{i}(\kappa)\right)=r_{1}+\cdots+r_{\kappa}+r(q-\kappa) .
\end{aligned}
$$

It is easy to verify that the numbers

$$
\begin{aligned}
l_{i}(\kappa) & =l_{i} & & (i=1, \ldots, \kappa), \\
m_{i}(\kappa) & =m_{i} & & (i=1, \ldots, \kappa), \\
l_{i}(\kappa) & =m_{i}(\kappa)=r / 2 & & (i>\kappa)
\end{aligned}
$$

satisfy (3.35), (3.36). The principal part of (3.16) corresponding to these numbers is a block diagonal matrix

$$
\left(\begin{array}{cc}
A^{0}(\kappa) & 0 \\
0 & -\lambda I
\end{array}\right)
$$

where $I$ is the $(q-\kappa) \times(q-\kappa)$ identity matrix. Taking the determinant of (3.37) we obtain (3.34).

Lemma 3.12. Let (3.28) be satisfied. Then the relations

$$
P_{r_{\kappa}}(\xi, \lambda)=(-\lambda)^{q-\kappa} \operatorname{det}\left(A^{0}(\kappa)(\xi)-\lambda E_{\kappa}\right),
$$


hold, where $E_{\kappa}$ is the $\kappa \times \kappa$ matrix whose element with indices $(\kappa, \kappa)$ equals 1 and the other elements are zero.

Proof. Attaining the weight $r_{\kappa}$ to $\lambda$ and repeating the argument of Lemma 3.11 we obtain that the principal part of (3.16) corresponding to this weight is equal to

$$
\left(\begin{array}{cc}
A^{0}(\kappa)(\xi)-\lambda E_{\kappa} & 0 \\
0 & -\lambda I
\end{array}\right)
$$

Taking the determinant of this matrix we obtain (3.38).

As a consequence of the relations (3.29), (3.30), (3.34) and (3.38) we obtain that under the validity of $(3.28)$ the definition of ellipticity with parameter is equivalent to the following conditions:

$$
\begin{array}{ll}
\operatorname{det} A^{0}(\kappa)(\xi) \neq 0 & (|\xi| \neq 0, \kappa=1,2, \ldots, q), \\
\operatorname{det}\left(A^{0}(\kappa)(\xi)-\lambda E_{\kappa}\right) \neq 0 & (|\xi| \neq 0,|\lambda| \neq 0, \lambda \in \mathcal{L}) .
\end{array}
$$

Now we consider the general case where some inequalities in (3.15) may be nonstrict. More precisely, suppose that

$$
r_{1}=\cdots=r_{t_{1}}>r_{t_{1}+1}=\cdots=r_{t_{2}}>\cdots>r_{t_{Q-1}+1}=\cdots=r_{t_{Q}} \geq 0
$$

and set

$$
\bar{r}_{1}=r_{t_{1}}>\bar{r}_{2}=r_{t_{2}}, \ldots, \bar{r}_{Q}=r_{t_{Q}} .
$$

We introduce a block structure in $A(\xi)$. For $\alpha, \beta=1, \ldots, Q$ we define the blocks

$$
A(\alpha, \beta)(\xi)=\left(A_{i j}(\xi)\right)_{\substack{i=t_{\alpha-1}+1, \ldots, t_{\alpha} \\ j=t_{\beta-1}+1, \ldots, t_{\beta}}}
$$

Accordingly, we write $A(\xi)$ in the form

$$
A(\xi)=(A(\alpha, \beta)(\xi))_{\alpha, \beta=1, \ldots, Q} .
$$

Replacing $A_{i j}$ by $A_{i j}^{0}$ we obtain the principal parts of the blocks which we denote by $A^{0}(\alpha, \beta)$.

We preserve the notation $E_{\kappa}$ for the block matrix whose right-corner block is the identity matrix and the other blocks are zero.

Definition 3.13. (KOZHEVNIKOV, see [K2]). We say that the matrix (3.16) has elliptic with parameter principal minors if for $\kappa=1, \ldots, Q$

$$
\operatorname{det} A^{0}(\kappa, \kappa)(\xi) \neq 0 \quad(|\xi| \neq 0),
$$

and if there exists a ray (1.1) such that

$$
\operatorname{det}\left(A^{0}(\kappa, \kappa)(\xi)-\lambda E_{\kappa}\right) \neq 0 \quad(|\lambda| \neq 0, \lambda \in \mathcal{L})
$$


Replacing in the arguments above the elements of $A$ by the corresponding blocks we obtain the main result of this section.

Theorem 3.14. Suppose that for the matrix (3.1) the conditions (3.2), (3.4), (3.5), and (3.42) hold. Then the following statements are equivalent:

(I) The matrix is elliptic with parameter (Definition 3.5).

(II) Inequality (3.21) holds (Proposition 3.7).

(III) The elements of the inverse matrix (3.23) satisfy the estimates (3.24), (3.25) (Proposition 3.10).

(IV) The KozHevnikov conditions hold (Definition 3.13).

\section{Ellipticity with parameter for systems in $\mathbb{R}^{n}$. The case of constant coefficients.}

In this section we consider the system of partial differential equations in $\mathbb{R}^{n}$

$$
A(D) u(x)-\lambda u(x)=f(x)
$$

where $u=\left(u_{1}, \ldots, u_{q}\right)$ and $f=\left(f_{1}, \ldots, f_{q}\right)$ belong to the function spaces which will be constructed below, and the symbol $A(\xi)$ satisfies the equivalent conditions of Theorem 3.14. Moreover, to simplify the presentation, we will suppose that the strong condition (3.28) holds. The unessential technical details connected with the replacement of scalar elements by square block matrices are left to the reader.

To formulate the statement we introduce the function spaces corresponding to the left - hand and right - hand side of (4.1). Set

$$
\begin{aligned}
& U=U\left(\mathbb{R}^{n}\right)=\prod_{j=1}^{q} H^{m_{j}}\left(\mathbb{R}^{n}\right), \\
& F=F\left(\mathbb{R}^{n}\right)=\prod_{j=1}^{q} H^{-l_{j}}\left(\mathbb{R}^{n}\right),
\end{aligned}
$$

where $H^{s}\left(\mathbb{R}^{n}\right)$ designates the standard Sobolev space. In these spaces we introduce parameter-dependent norms, and we denote by $U(\lambda)$ and $F(\lambda)$ the spaces $U$ and $F$, respectively, endowed with these norms. We set

$$
\|u, U(\lambda)\|^{2}=\sum_{j=1}^{q}\left\|\left(|D|^{2 r_{j}}+|\lambda|^{2}\right)^{\frac{m_{j}}{2 r_{j}}} u_{j}\right\|^{2},
$$

where $\|\cdot\|$ denotes the norm in $L_{2}\left(\mathbb{R}^{n}\right)$. As in Section 3 we formally set $m_{i} / r_{i}=1$ for $r_{i}=0$. The corresponding term on the right-hand side of (4.3) is equal to $\left(1+|\lambda|^{2}\right)\left\|u_{i}\right\|^{2}$.

Defining the norm in $F$ we shall distinguish the cases $r_{q}>0$ and $r_{q}=0$. In the first case we set

$$
\|f, F(\lambda)\|^{2}=\sum_{j=1}^{q}\left\|\left(|D|^{2 r_{j}}+|\lambda|^{2}\right)^{-\frac{l_{j}}{2 r_{j}}} f_{j}\right\|^{2} .
$$


In the general case we suppose that

$$
r_{1} \geq \cdots \geq r_{q^{\prime}}>r_{q^{\prime}+1}=\cdots=r_{q}=0
$$

Then we set

$$
\|f, F(\lambda)\|^{2}=\sum_{j=1}^{q^{\prime}}\left\|\left(|D|^{2 r_{j}}+|\lambda|^{2}\right)^{-\frac{l_{j}}{2 r_{j}}} f_{j}\right\|^{2}+\sum_{j=q^{\prime}+1}^{q}\left\|f_{j}\right\|^{2} .
$$

Theorem 4.1. For the matrix $A(\xi)$ satisfying (3.2), (3.4), (3.5) and (3.15) the following statements are equivalent:

(A) The matrix $A(\xi)-\lambda I$ satisfies the equivalent conditions of Theorem 3.14.

(B) There exists a ray (1.1) and a $\rho_{0}>0$ such that for $\lambda \in \mathcal{L},|\lambda| \geq \rho_{0}$, and for arbitrary $f \in F(\lambda)$ the equation (4.1) has a unique solution $u \in U(\lambda)$, and the inequality

$$
\|u, U(\lambda)\| \leq K\|f, F(\lambda)\|
$$

holds with $K$ independent of $\lambda$.

$\left(\mathrm{B}_{0}\right)$ There exists a ray $(1.1)$ and a $\rho_{0}>0$ such that for $u_{j} \in H^{\infty}\left(\mathbb{R}^{n}\right), j=1, \ldots, q$, the a priori estimate

$$
\|u, U(\lambda)\| \leq K\|(A(D)-\lambda I) u, F(\lambda)\|, \quad\left(\lambda \in \mathcal{L},|\lambda| \geq \rho_{0}\right)
$$

holds with $K$ independent of $\lambda$.

Pr o of . $(A) \Rightarrow(B)$. Applying the Fourier transform to both sides of (4.1) for large $|\lambda|$, we obtain

$$
\widehat{u}(\xi)=G(\xi, \lambda) \widehat{f}(\xi),
$$

where $\widehat{u}(\xi)$ and $\widehat{f}(\xi)$ denote the Fourier transform of $u$ and of $f$, respectively. At first, we suppose that all $r_{j}$ are strictly positive. Then, according to the Cauchy inequality,

$$
\left|\widehat{u}_{i}(\xi)\right|^{2} \leq \sum_{j=1}^{q}\left|G_{i j}(\xi, \lambda)\right|^{2}\left(|\xi|^{2 r_{j}}+|\lambda|^{2}\right)^{\frac{l_{j}}{r_{j}}} \sum_{j=1}^{q}\left(|\xi|^{2 r_{j}}+|\lambda|^{2}\right)^{-\frac{l_{j}}{r_{j}}}\left|\widehat{f}_{j}(\xi)\right|^{2}
$$

If we multiply both sides by $\left(|\xi|^{2 r_{i}}+|\lambda|^{2}\right)^{m_{i} / r_{i}}$ and take into account inequalities (3.26), we obtain the estimate

$$
\sum\left(|\xi|^{2 r_{i}}+|\lambda|^{2}\right)^{m_{i} / r_{i}}\left|\widehat{u}_{i}(\xi)\right|^{2} \leq K^{2} \sum\left(|\xi|^{2 r_{j}}+|\lambda|^{2}\right)^{-l_{j} / r_{j}}\left|\widehat{f}_{j}(\xi)\right|^{2}
$$

Integrating both sides with respect to $\xi$, we obtain (4.7).

In the case (4.5) the above argument should be slightly modified. For $i \leq q^{\prime}$ inequalities (4.9) have sense because (as it was mentioned in Section 3) estimates (3.26) can be extended to the case when one of the numbers $r_{i}, r_{j}$ is zero but the other is 
positive. In the case $i>q^{\prime}$ we use the inequality

$$
\begin{aligned}
\left(1+|\lambda|^{2}\right)\left|\widehat{u}_{i}(\xi)\right|^{2} \leq & \left(\sum_{j=1}^{q^{\prime}}\left|G_{i j}(\xi, \lambda)\right|^{2}\left(|\xi|^{2 r_{j}}+|\lambda|^{2}\right)^{l_{j} / r_{j}}\left(1+|\lambda|^{2}\right)+\right. \\
& \left.+\sum_{j=q^{\prime}+1}^{q}\left|G_{i j}(\xi, \lambda)\right|^{2}\left(1+|\lambda|^{2}\right)\right) \\
& \times\left(\sum_{j=1}^{q^{\prime}}\left(|\xi|^{2 r_{j}}+|\lambda|^{2}\right)^{-l_{j} / r_{j}}\left|\widehat{f}_{j}(\xi)\right|^{2}+\sum_{j=q^{\prime}+1}^{q}\left|\widehat{f}_{j}(\xi)\right|^{2}\right) .
\end{aligned}
$$

It follows from (3.26) and (3.27) that the first bracket on the right-hand side can be estimated by a constant independent of $\xi$ and $\lambda$.

Summing up, we have proved the solvability of (4.1) for $|\lambda| \geq \rho_{0}$ and the validity of (4.7).

$(\mathrm{B}) \Rightarrow\left(\mathrm{B}_{0}\right) \Rightarrow(\mathrm{A})$. The first implication is trivial, the second follows from the lemmas below.

Lemma 4.2. Suppose that (4.8) holds for $\lambda \in \mathcal{L}$ with $|\lambda| \geq \rho_{0}$. Then for $\kappa=$ $1, \ldots, q, r_{\kappa}>0$ we have

$$
\begin{gathered}
\sum_{j=1}^{\kappa}\left\||D|^{m_{j}} u_{j}\right\|^{2} \leq K^{2} \sum_{j=1}^{\kappa}\left\|\sum_{k=1}^{\kappa}|D|^{-l_{j}} A_{j k}^{0}(D) u_{k}\right\|^{2}, \\
u_{j} \in H^{\infty}\left(\mathbb{R}^{n}\right), \quad j=1, \ldots, \kappa, \\
\sum_{j=1}^{\kappa-1}\left\||D|^{m_{j}} u_{j}\right\|^{2}+\left\|\left(|D|^{2 r_{\kappa}}+|\lambda|^{2}\right)^{\frac{m_{\kappa}}{2 r_{\kappa}}} u_{\kappa}\right\|^{2} \\
\leq K^{2}\left(\sum_{j=1}^{\kappa-1}\left\|\sum_{k=1}^{\kappa}|D|^{-l_{j}} A_{j k}^{0} u_{k}\right\|^{2}\right. \\
\left.+\left\|\left(|D|^{2 r_{\kappa}}+|\lambda|^{2}\right)^{-\frac{l_{\kappa}}{2 r_{\kappa}}}\left(\sum_{k=1}^{\kappa} A_{\kappa k}^{0} u_{k}-\lambda u_{\kappa}\right)\right\| \|^{2}\right), \\
u_{j} \in H^{\infty}\left(\mathbb{R}^{n}\right), \quad j=1, \ldots, \kappa, \lambda \in \mathcal{L}, \quad|\lambda|>0 .
\end{gathered}
$$

Lemma 4.3. (i) Suppose that (4.10) holds. Then

$$
\operatorname{det} A^{0}(\kappa)(\xi) \neq 0 \quad\left(\xi \in \mathbb{R}^{n},|\xi|>0\right) .
$$

(ii) Suppose that (4.11) holds. Then

$$
\operatorname{det}\left(A^{0}(\kappa)(\xi)-\lambda E_{\kappa}\right) \neq 0 \quad\left((\lambda, \xi) \in \mathcal{L} \times \mathbb{R}^{n},|\lambda|>0,|\xi|>0\right) .
$$


Pro of of Lemma 4.2. We begin with deriving (4.10). We replace $\lambda$ and $u(x)$ in (4.8) by $\lambda \rho^{r}$ and

$$
u_{\rho}(x)=\left(u_{1 \rho}(x), \ldots, u_{q \rho}(x)\right), \quad u_{j \rho}(x)=\rho^{\frac{n}{2}-\mu_{j}(\kappa)} u_{j}(\rho x),
$$

where the numbers $r$ and $\mu_{j}(\kappa)$ are chosen in the following way

$$
\begin{gathered}
r_{\kappa}>r>r_{\kappa+1}, \\
\mu_{j}(\kappa)=m_{j}, \quad j=1, \ldots, \kappa, \\
\mu_{j}(\kappa)=\frac{r}{r_{j}} m_{j}, \quad j \geq \kappa+1 .
\end{gathered}
$$

Put

$$
I_{j \rho}^{2}=\left\|\left(|D|^{2 r_{j}}+|\lambda|^{2} \rho^{2 r}\right)^{\frac{m_{j}}{2 r_{j}}} u_{j \rho}\right\|^{2} .
$$

Direct calculations show that

$$
\widehat{u}_{j \rho}(\xi)=\rho^{-n / 2-\mu_{j}(\kappa)} \widehat{u}_{j}(\xi / \rho) .
$$

Now we obtain

$$
\begin{aligned}
I_{j \rho}^{2} & =\int \rho^{-n-2 \mu_{j}(\kappa)}\left(|\xi|^{2 r_{j}}+|\lambda|^{2} \rho^{2 r}\right)^{\frac{m_{j}}{r_{j}}}\left|\widehat{u}_{j}(\xi / \rho)\right|^{2} d \xi \\
& =\int \rho^{-2 \mu_{j}(\kappa)}\left(\rho^{2 r_{j}}|\xi|^{2 r_{j}}+\rho^{2 r}|\lambda|^{2}\right)^{\frac{m_{j}}{r_{j}}}\left|\widehat{u}_{j}(\xi)\right|^{2} d \xi .
\end{aligned}
$$

If $j \leq \kappa$, then $\mu_{j}(\kappa)=m_{j}$ and

$$
I_{j \rho}^{2}=\int\left(|\xi|^{2 r_{j}}+\rho^{-2\left(r-r_{j}\right)}|\lambda|^{2}\right)^{\frac{m_{j}}{r_{j}}}\left|\widehat{u}_{j}(\xi)\right|^{2} d \xi \longrightarrow \int|\xi|^{2 m_{j}}\left|\widehat{u}_{j}(\xi)\right|^{2} d \xi=\left\||D|^{m_{j}} u_{j}\right\|^{2},
$$

for $\rho \rightarrow+\infty$ (we used the inequalities $r_{j} \geq r_{\kappa}>r$ ).

If $j>\kappa$, then

$$
I_{j \rho}^{2}=\int\left(\rho^{-2\left(r-r_{j}\right)}|\xi|^{2 r_{j}}+|\lambda|^{2}\right)^{\frac{m_{j}}{r_{j}}}\left|\widehat{u}_{j}(\xi)\right|^{2} d \xi \longrightarrow|\lambda|^{\frac{2 m_{j}}{r_{j}}}\left\|u_{j}\right\|^{2}, \quad \rho \longrightarrow+\infty .
$$

Summing up we see that for $\rho \rightarrow+\infty$

$$
\left\|u_{\rho}, U\left(\lambda \rho^{r}\right)\right\|^{2} \longrightarrow \sum_{j=1}^{\kappa}\left\||D|^{m_{j}} u_{j}\right\|^{2}+\sum_{j=\kappa+1}^{q}|\lambda|^{\frac{2 m_{j}}{r_{j}}}\left\|u_{j}\right\|^{2} .
$$

Now we consider

$$
\begin{aligned}
J_{j \rho}^{2} & =\left\|\left(|D|^{2 r_{j}}+\rho^{2 r}|\lambda|^{2}\right)^{-\frac{l_{j}}{2 r_{j}}}\left(\sum_{k=1}^{q} A_{j k}(D) u_{k \rho}-\lambda \rho^{r} u_{j \rho}\right)\right\|^{2} \\
& =\int\left(\rho^{2 r_{j}}|\xi|^{2 r_{j}}+\rho^{2 r}|\lambda|^{2}\right)^{-\frac{l_{j}}{r_{j}}}\left|\sum_{k=1}^{q} A_{j k}(\rho \xi) \rho^{-\mu_{k}(\kappa)} \widehat{u}_{k}(\xi)-\lambda \rho^{r-\mu_{j}(\kappa)} \widehat{u}_{j}(\xi)\right|^{2} d \xi .
\end{aligned}
$$


Note that

$$
A_{j k}(\rho \xi)=\rho^{l_{j}+m_{k}}\left(A_{j k}^{0}(\xi)+O\left(\rho^{-1}\right)\right) .
$$

For $j=1, \ldots, \kappa$ the limit of $J_{j \rho}^{2}$ as $\rho \rightarrow+\infty$ is equal to

$$
\int|\xi|^{-2 l_{j}}\left|\sum_{k=1}^{\kappa} A_{j k}^{0}(\xi) \widehat{u}_{k}(\xi)\right|^{2} d \xi=\left\||D|^{-l_{j}} \sum_{k=1}^{\kappa} A_{j k}^{0}(D) u_{k}\right\|^{2} .
$$

For $j>\kappa$ the limit of $J_{j \rho}^{2}$ as $\rho \rightarrow+\infty$ is equal to

$$
|\lambda|^{2 m_{j} / r_{j}}\left\|u_{j}\right\|^{2}
$$

Thus, we have proved the inequality

$$
\begin{aligned}
& \sum_{j=1}^{\kappa}\left\||D|^{m_{j}} u_{j}\right\|^{2}+\sum_{j=\kappa+1}^{q}|\lambda|^{2 m_{j} / r_{j}}\left\|u_{j}\right\|^{2} \\
\leq & K^{2}\left(\sum_{j=1}^{\kappa}\left\||D|^{-l_{j}} \sum_{k=1}^{\kappa} A_{j k}^{0}(D) u_{k}\right\|^{2}+\sum_{j=\kappa+1}^{q}|\lambda|^{2 m_{j} / r_{j}}\left\|u_{j}\right\|^{2}\right) .
\end{aligned}
$$

Setting $u_{\kappa+1}(x)=\cdots=u_{q}(x) \equiv 0$ we obtain (4.10).

To prove (4.11) we make the same change in (4.12) with (4.13) replaced by

$$
r_{\kappa}=r \text {. }
$$

Repeating the above arguments almost literally we prove (4.11).

Pr o of of Lemma 4.3. (i) To simplify the notation we suppose that $\kappa=q$. Assume that (i) does not hold, i. e., there exists a $\xi^{0} \neq 0$ such that

$$
\operatorname{det} A^{0}\left(\xi^{0}\right)=0 \text {. }
$$

Then there exists a vector $h=\left(h_{1}, \ldots, h_{q}\right) \in \mathbb{C}^{q},|h| \neq 0$, such that

$$
A^{0}\left(\xi^{0}\right) h=0 \text {. }
$$

Now we take $u_{\rho}(x)=\left(u_{1 \rho}(x), \ldots, u_{q \rho}(x)\right)$, where

$$
u_{j \rho}(x)=\phi(x) \exp \left(i \rho \xi^{0} \cdot x\right) \rho^{-m_{j}} h_{j} \quad(j=1, \ldots, q),
$$

and $\phi(x) \in \mathcal{D}\left(\mathbb{R}^{n}\right)$. We substitute (4.17) in (4.10) and show that

$$
\begin{gathered}
\sum_{j=1}^{q}\left\||D|^{m_{j}} u_{\rho j}\right\|^{2}=O(1) \quad(\rho \rightarrow+\infty), \\
\sum_{i=1}^{q}\left\||D|^{-l_{i}} \sum_{j=1}^{q} A_{i j}^{0}(D) u_{\rho j}\right\|=o(1) \quad(\rho \rightarrow+\infty) .
\end{gathered}
$$


Since (4.18), (4.19) contradict (4.10), we prove our statement.

Note that

$$
\widehat{u}_{\rho j}(\xi)=\rho^{-m_{j}} \widehat{\phi}\left(\xi-\rho \xi^{0}\right) h_{j} .
$$

Then as $\rho \rightarrow+\infty$

$$
\begin{aligned}
\sum_{j=1}^{q}\left\||D|^{m_{i}} u_{\rho j}\right\|^{2} & =\sum_{j=1}^{q} \rho^{-2 m_{j}}\left|h_{j}\right|^{2} \int|\xi|^{2 m_{j}}\left|\widehat{\phi}\left(\xi-\rho \xi^{0}\right)\right|^{2} d \xi \\
& =\sum_{j=1}^{q} \rho^{-2 m_{j}}\left|h_{j}\right|^{2} \int\left|\xi+\rho \xi^{0}\right|^{2 m_{j}}|\widehat{\phi}(\xi)|^{2} d \xi \\
& \longrightarrow \sum_{j=1}^{q}\left|\xi^{0}\right|^{2 m_{j}}\left|h_{j}\right|^{2}\|\phi\|^{2} .
\end{aligned}
$$

Since $\left|\xi^{0}\right| \neq 0$ and $\sum\left|h_{j}\right|^{2} \neq 0$, we obtain (4.18).

Now we calculate the limit of the left - hand side of (4.19) as $\rho \rightarrow+\infty$. We have

$$
\begin{aligned}
\left\||D|^{-l_{i}} \sum_{j=1}^{q} A_{i j}^{0}(D) u_{\rho j}\right\|^{2} & =\int|\xi|^{-2 l_{i}}\left|\widehat{\phi}\left(\xi-\rho \xi^{0}\right)\right|^{2}\left|\sum_{j=1}^{q} A_{i j}^{0}(\xi) \rho^{-m_{j}} h_{j}\right|^{2} d \xi \\
& =\int|\widehat{\phi}(\xi)|^{2}\left|\xi+\rho \xi^{0}\right|^{-2 l_{i}}\left|\sum_{j=1}^{q} A_{i j}^{0}\left(\xi+\rho \xi^{0}\right) \rho^{-m_{j}} h_{j}\right|^{2} d \xi \\
& =\int_{|\xi|>\varepsilon \rho} \ldots+\int_{|\xi|<\varepsilon \rho} \ldots \\
& =J_{j \rho}^{\prime}+J_{j \rho}^{\prime \prime} .
\end{aligned}
$$

Since the function $\widehat{\phi}(\xi)$ decreases faster than any power of $|\xi|$, we have for arbitrary $N \in \mathbb{N}$

$$
J_{i \rho}^{\prime}=O\left(\rho^{-N}\right) \quad(\rho \rightarrow+\infty) .
$$

Before estimating $J_{j \rho}^{\prime \prime}$ we note that according to (4.16)

$$
\begin{aligned}
\sum_{j} A_{i j}^{0}\left(\xi+\rho \xi^{0}\right) \rho^{-m_{j}} h_{j} & =\rho^{l_{i}} \sum_{j} A_{i j}^{0}\left(\xi^{0}+\rho^{-1} \xi\right) h_{j} \\
& =\rho^{l_{i}} \sum_{|\alpha|>0} \sum_{j} \frac{1}{\alpha !}\left(\partial^{\alpha} A_{i j}^{0}\right)\left(\xi^{0}\right) h_{j} \rho^{-|\alpha|} \xi^{\alpha} \\
& =\rho^{l_{i}} \sum_{|\alpha|>0} c_{\alpha} \rho^{-|\alpha|} \xi^{\alpha}
\end{aligned}
$$

Then

$$
J_{j \rho}^{\prime \prime}=\int_{|\xi| \leq \varepsilon \rho}|\widehat{\phi}(\xi)|^{2}\left|\xi^{0}+\rho^{-1} \xi\right|^{-2 l_{i}}\left|\sum_{|\alpha|>0} c_{\alpha} \rho^{-|\alpha|} \xi^{\alpha}\right|^{2} d \xi
$$


For $|\xi|<\varepsilon \rho$ we have

$$
\left|\xi^{0}+\rho^{-1} \xi\right|>\left|\xi^{0}\right|-\rho^{-1}|\xi|>\left|\xi^{0}\right|-\varepsilon>\frac{1}{2}\left|\xi^{0}\right|
$$

if $\varepsilon$ is small enough. Then

$$
J_{j \rho}^{\prime \prime} \leq \mathrm{const} \sum_{|\beta| \geq 1} \rho^{-2|\beta|}\left\|D^{\beta} \phi\right\|^{2} \longrightarrow 0 \quad(\rho \rightarrow+\infty) .
$$

(ii) As above we assume that $\kappa=q$. We suppose that there exist $\xi^{0} \in \mathbb{R}^{n},\left|\xi^{0}\right| \neq$ $0, \lambda^{0} \in \mathcal{L},\left|\lambda^{0}\right| \neq 0$ and $h \in \mathbb{C}^{q},|h| \neq 0$, such that

$$
\left(A^{0}\left(\xi^{0}\right)-\lambda^{0} E_{q}\right) h=0 .
$$

We replace $\lambda$ and $u_{j}(x)$ in (4.11) by $\lambda^{0} \rho^{r_{\kappa}}$ and $u_{j \rho}(x)$, respectively. Repeating the above arguments we obtain the contradiction which proves our statement.

\section{Ellipticity with parameter for systems with variable coeffi - cients}

\subsection{Plan of the proof of the solvability in $\mathbb{R}^{n}$}

We begin with the investigation of unique solvability (for large $\lambda \in \mathcal{L}$ ) of the system

$$
A(x, D) u(x)-\lambda u(x)=f(x)
$$

in $\mathbb{R}^{n}$. Here

$$
A(x, \xi)=\left(A_{i j}(x, \xi)\right)_{i, j=1, \ldots, q}, \quad A_{i j}(x, \xi)=\sum a_{i j \alpha}(x) \xi^{\alpha}
$$

where the coefficients are $C^{\infty}$ and are constant at infinity. More precisely,

$$
a_{i j \alpha}(x)=a_{i j \alpha}+a_{i j \alpha}^{\prime}(x), \quad a_{i j \alpha}^{\prime}(x) \in \mathcal{D} .
$$

Set

$$
\alpha_{i j}=\sup _{x \in \mathbb{R}^{n}} \operatorname{deg} A_{i j}(x, \xi) .
$$

As in Section 3 we define the numbers $l_{1}, \ldots, l_{q}, m_{1}, \ldots, m_{q}$ such that the relations (3.4), (3.5) are satisfied. We suppose, in addition, that we can choose nonnegative numbers $l_{1}, \ldots, m_{q}$, and they are indexed in such way that inequalities (3.14) and (3.15) hold. To simplify the notation we suppose that (3.28) holds.

Using the numbers $l_{1}, \ldots, m_{q}$ we define the principal part of the symbol (5.2) in the standard way:

$$
A^{0}(x, \xi)=\left(A_{i j}^{0}(x, \xi)\right)_{i, j=1, \ldots, q}, \quad A_{i j}^{0}(x, \xi)=\sum_{|\alpha|=l_{i}+m_{j}} a_{i j \alpha}(x) \xi^{\alpha} .
$$


Definition 5.1. The symbol (5.2) is called elliptic with parameter if the following conditions are satisfied:

(i) For each $x^{0} \in \mathbb{R}^{n}$ the Newton polygon $N\left(P\left(x^{0}\right)\right)$ of the polynomial $P\left(x^{0}, \xi, \lambda\right)$, where

$$
P(x, \xi, \lambda)=\operatorname{det}(A(x, \xi)-\lambda I),
$$

contains the points (3.19).

(ii) There exists a ray (1.1) and positive $d, \rho_{0}$ such that the estimate

$$
d \Xi_{P}(\xi, \lambda) \leq|P(x, \xi, \lambda)| \text { for }(x, \xi) \in \mathbb{R}^{n} \times \mathbb{R}^{n}, \quad \lambda \in \mathcal{L},|\lambda| \geq \rho_{0},
$$

holds.

Remark 5.2. (Cf. Remark 3.6). For each $x^{0} \in \mathbb{R}^{n}$ the polygon $N\left(P\left(x^{0}\right)\right)$ belongs to the convex hull of the points (3.19). Thus, according to (i) all the polygons $N\left(P\left(x^{0}\right)\right)$ coincide with the convex hull of the points (3.19) and are independent of $x^{0}$. Due to this fact the notation on the left-hand side (5.5) is correct.

Theorem 5.3. For the system (5.1) with coefficients of the form (5.3) the following statements are equivalent:

(A) The symbol $A(x, \xi)-\lambda I$ satisfies the conditions (i), (ii) of Definition 5.1.

(B) There exists a ray (1.1) and a number $\rho_{0}>0$ such that for $\lambda \in \mathcal{L}$ with $|\lambda| \geq \rho_{0}$ and for arbitrary $f \in F(\lambda)$ the equation (5.1) has a unique solution $u \in U(\lambda)$, and the estimate

$$
\|u, U(\lambda)\| \leq K\|f, F(\lambda)\|
$$

holds with $K$ independent of $\lambda$.

$\left(\mathrm{B}_{0}\right)$ There exists a ray $(1.1)$ and $\rho_{0}>0$ such that for $u_{j} \in H^{\infty}\left(\mathbb{R}^{n}\right), j=1, \ldots, q$, the a priori estimate

$$
\|u, U(\lambda)\| \leq K\|(A(x, D)-\lambda I) u, F(\lambda)\| \quad\left(\lambda \in \mathcal{L},|\lambda| \geq \rho_{0}\right)
$$

holds with $K$ independent of $\lambda$.

Proof. (A) $\Rightarrow(B)$. Set

$$
G(x, \xi, \lambda)=(A(x, \xi)-\lambda I)^{-1}=\left(G_{i j}(x, \xi, \lambda)\right)_{i, j=1, \ldots, q}
$$

and consider the pseudodifferential matrix operator $G(x, D, \lambda)$ with this symbol. The proof of the solvability of (5.1) is traditional and is based on

Proposition 5.4. Suppose that the conditions of Definition 5.1 are satisfied and the matrix $G(x, D, \lambda)$ is given by (5.8).

(i) The operator

$$
F(\lambda) \longrightarrow U(\lambda), \quad f \longmapsto G(x, D, \lambda) f,
$$

is continuous for $|\lambda| \geq \rho_{0}, \lambda \in \mathcal{L}$, and its norm is uniformly bounded for such $\lambda$. 
(ii) The norm of the operator

$$
F(\lambda) \longrightarrow F(\lambda), \quad f \longmapsto(A(x, D)-\lambda I) G(x, D, \lambda) f-f \text {, }
$$

tends to zero as $\lambda \in \mathcal{L},|\lambda| \rightarrow \infty$.

(iii) The norm of the operator

$$
U(\lambda) \longrightarrow U(\lambda), \quad u \longrightarrow G(x, D, \lambda)(A(x, D)-\lambda I) u-u
$$

tends to zero as $\lambda \in \mathcal{L},|\lambda| \rightarrow \infty$.

Assuming that the proposition is proved we complete the derivation of the implication $(\mathrm{A}) \Rightarrow(\mathrm{B})$.

Solvability of Equation (5.1). We seek the solution in the form $u=G(x, D, \lambda) g$ with $g \in F(\lambda)$. According to Proposition 5.4(i), $u \in U(\lambda)$. Inserting in (5.1) the expression for $u$, we obtain an equation for $g$ given by

$$
g+[(A-\lambda I) G-I] g=f .
$$

According to Proposition 5.4 (ii), there exists $\rho_{1}>0$ such that the norm of the operator in the square brackets is less than 1 for $\lambda \in \mathcal{L},|\lambda| \geq \rho_{1}$. Then equation (4.11) has a unique solution $g \in F(\lambda)$.

Proof of the estimate (5.6). Applying the operator $G(x, D, \lambda)$ to both sides of $(5.1)$, we obtain

$$
u+[G(A-\lambda I)-I] u=G f .
$$

Therefore, taking into account Proposition 5.4, we obtain

$$
\begin{aligned}
\|u, U(\lambda)\| & \leq\|G f, U(\lambda)\|+\|G(A-\lambda I) u-u, U(\lambda)\| \\
& \leq K\|f, F(\lambda)\|+\varepsilon(\lambda)\|u, U(\lambda)\| .
\end{aligned}
$$

According to Proposition 5.4 (iii), $\varepsilon(\lambda) \rightarrow 0(|\lambda| \rightarrow \infty)$. Hence there exists a $\rho_{1}$ such that $\varepsilon(\lambda)<1$ as $\lambda \in \mathcal{L},|\lambda|>\rho_{1}$, and we obtain (5.6). The uniqueness for (5.1) follows from this estimate.

$(\mathrm{B}) \Rightarrow\left(\mathrm{B}_{0}\right) \Rightarrow(\mathrm{A})$. The first statement is trivial. The proof of the second implication is a slight modification of the analogous proof in Theorem 4.1 and is based on

Proposition 5.5. Suppose that (5.7) holds. Let $x^{0} \in \mathbb{R}^{n}$ be arbitrary and $A^{0}(D)=$ $A^{0}\left(x^{0}, D\right)$. Then the estimates $(4.10)$, (4.11) are valid for $\kappa=1, \ldots, q\left(r_{\kappa}>0\right)$ with constant $K$ independent of $x^{0}$.

It follows from the Proposition and Lemma 4.3 that the operator $A\left(x^{0}, D\right)$ satisfies the equivalent conditions of Theorem 4.1. Hence (5.5) holds for each $x^{0} \in \mathbb{R}^{n}$ with $d=d\left(x^{0}\right)$. Since $d\left(x^{0}\right)$ depends only on the constant $K$ in (4.10), (4.11) and $K$ is independent on $x^{0}$, the constant $d\left(x^{0}\right)$ is also independent on $x^{0}$, and condition $(\mathrm{A})$ holds. 


\subsection{Proof of Proposition 5.4}

(i) According to the definition of the norms (4.3), (4.4) and (4.6), the $F(\lambda) \rightarrow U(\lambda)$ boundedness of the operator (5.9) is equivalent to the $L_{2} \rightarrow L_{2}$ boundedness of the operators

$$
\left(|D|^{2 r_{i}}+|\lambda|^{2}\right)^{\frac{m_{i}}{2 r_{i}}} G_{i j}(x, D, \lambda)\left(|D|^{2 r_{j}}+|\lambda|^{2}\right)^{\frac{l_{j}}{2 r_{j}} \vartheta\left(r_{j}\right)}
$$

where (in correspondence with (4.6)) $\vartheta\left(r_{j}\right)=1$ for $r_{j}>0$ and $\vartheta\left(r_{j}\right)=0$ for $r_{j}=0$.

Due to standard estimates for the norms of pseudodifferential operators it suffices to establish the following estimates for $D_{x}^{\alpha} G_{i j}$ :

$$
\left(|\xi|^{r_{i}}+|\lambda|\right)^{\frac{m_{i}}{r_{i}}}\left(|\xi|^{r_{j}}+|\lambda|\right)^{\frac{l_{j}}{r_{j}} \vartheta\left(r_{j}\right)}\left|D_{x}^{\alpha} G_{i j}(x, \xi, \lambda)\right| \leq C_{i j \alpha}(\lambda)
$$

where the functions $C_{i j \alpha}(\lambda)$ are independent of $x$ and uniformly bounded as $\lambda \in \mathcal{L},|\lambda| \geq \rho_{0}$. The estimates (5.14) can be directly derived from the estimates (cf. $(3.24),(3.25))$

$$
\begin{aligned}
& \left|D_{x}^{\alpha} G_{i j}(x, \xi, \lambda)\right| \leq C_{i j \alpha}(1+|\xi|)^{l_{i}+m_{j}}\left(|\xi|^{r_{i}}+|\lambda|\right)^{-1}\left(|\xi|^{r_{j}}+|\lambda|\right)^{-1}, \quad i \neq j \\
& \left|D_{x}^{\alpha} G_{i i}(x, \xi, \lambda)\right| \leq C_{i i \alpha}\left(|\xi|^{r_{i}}+|\lambda|\right)^{-1}
\end{aligned}
$$

Indeed, in view of (5.15), (5.16) the left-hand side of (5.14) for $|\lambda| \geq \rho_{0}$ can be estimated by

$$
\begin{aligned}
& C_{i j \alpha}(1+|\xi|)^{l_{i}+m_{j}}\left(|\xi|^{r_{i}}+|\lambda|\right)^{-\frac{l_{i}}{r_{i}}}\left(|\xi|^{r_{j}}+|\lambda|\right)^{-\frac{m_{j}}{r_{j}}} \\
\leq & C_{i j \alpha}(1+|\xi|)^{l_{i}+m_{j}}\left(|\xi|^{r_{i}}+\rho_{0}\right)^{-\frac{l_{i}}{r_{i}}}\left(|\xi|^{r_{j}}+\rho_{0}\right)^{-\frac{m_{j}}{r_{j}}} \\
\leq & C_{i j \alpha}\left(\rho_{0}\right) .
\end{aligned}
$$

The estimates (5.15), (5.16) for $\alpha=0$ with $C_{i j \sigma}(\lambda)$ independent of $\lambda$ for $|\lambda| \geq \rho_{0}$ can be obtained by a repetition of the proof of Proposition 3.10 and are based on the representation

$$
G_{i j}(x, \xi, \lambda)=P^{-1}(x, \xi, \lambda) \operatorname{det}\left((A(x, \xi)-\lambda I)^{j i}\right)
$$

From the rule of calculation of determinants we easily derive for an arbitrary multiindex $\gamma$ :

$$
\begin{aligned}
\left|D_{x}^{\gamma} \operatorname{det}\left((A(x, \xi)-\lambda I)^{j i}\right)\right| & \leq C_{i j \gamma}(1+|\xi|)^{l_{i}+m_{j}} \prod_{s \neq i, j}\left(|\xi|^{r_{s}}+|\lambda|\right), \\
\left|D_{x}^{\gamma} \operatorname{det}\left((A(x, \xi)-\lambda I)^{i i}\right)\right| & \leq C_{i i \gamma} \prod_{s \neq i}\left(|\xi|^{r_{s}}+|\lambda|\right) .
\end{aligned}
$$

If we prove that

$$
\left|D_{x}^{\beta} P^{-1}(x, \xi, \lambda)\right| \leq C_{\beta} \prod\left(|\xi|^{r_{s}}+|\lambda|\right)^{-1}
$$


the desired estimates (5.15), (5.16) follow from the Leibniz rule

$$
D_{x}^{\alpha} G_{i j}(x, \xi, \lambda)=\sum_{\beta+\gamma=\alpha} \frac{\alpha !}{\beta ! \gamma !} D_{x}^{\beta} P^{-1}(x, \xi, \lambda) D_{x}^{\gamma} \operatorname{det}\left((A(x, \xi)-\lambda I)^{j i}\right) .
$$

Now we derive (5.20). For $\beta=0$ the estimate follows from (5.5) and the equivalence of $\Xi_{P}(\xi, \lambda)$ and $\prod\left(|\xi|^{r_{s}}+|\lambda|\right)$. For $|\beta| \geq 1$ the left - hand side of (5.20) is the absolute value of a linear combination of expressions of the form

$$
\frac{D^{\beta^{1}} P(x, \xi, \lambda)}{P(x, \xi, \lambda)} \cdots \frac{D^{\beta^{k}} P(x, \xi, \lambda)}{P(x, \xi, \lambda)} P^{-1}(x, \xi, \lambda) .
$$

It follows from the definition of the Newton polygon that

$$
\left|D_{x}^{\beta} P(x, \xi, \lambda)\right| \leq K_{\beta} \Xi_{P}(\xi, \lambda) .
$$

Combining this estimate with (5.5), we estimate the expressions in (5.21) by the righthand side of (5.20).

(ii) Direct calculations show that $(A(x, D)-\lambda I) G(x, D, \lambda)-I$ is a matrix pseudodifferential operator with the symbol

$$
T(x, \xi, \lambda)=\sum_{|\alpha|>0} \frac{1}{\alpha !} \partial_{\xi}^{\alpha} A(x, \xi) D_{x}^{\alpha} G(x, \xi, \lambda) .
$$

In view of the above argument our statement will be proved if we establish the estimates of the $x$-derivatives of symbols (5.22):

$$
\left(|\xi|^{r_{i}}+|\lambda|\right)^{-\frac{l_{i}}{r_{i}} \vartheta\left(r_{i}\right)}\left(|\xi|^{r_{j}}+|\lambda|\right)^{-\frac{l_{j}}{r_{j}} \vartheta\left(r_{j}\right)}\left|D_{x}^{\beta} T_{i j}(x, \xi, \lambda)\right| \leq \varepsilon_{i j \beta}(\lambda),
$$

where $\varepsilon_{i j \beta}(\lambda) \rightarrow 0$ as $|\lambda| \rightarrow \infty$. According to the definition (5.22) of the matrix $T(x, \xi, \lambda)=\left(T_{i j}(x, \xi, \lambda)\right)_{i, j}$ we have

$$
T_{i j}(x, \xi, \lambda)=\sum_{|\alpha| \geq 1} \sum_{k=1}^{q} \frac{1}{\alpha !} \partial_{\xi}^{\alpha} A_{i k}(x, \xi) D_{x}^{\alpha} G_{k j}(x, \xi, \lambda) .
$$

Using (5.15), (5.16) and the obvious estimates

$$
\left|\partial_{\xi}^{\alpha} A_{i k}(x, \xi)\right| \leq \operatorname{const}(1+|\xi|)^{l_{i}+m_{k}-|\alpha|},
$$

we obtain

$$
\begin{aligned}
\left|T_{i j}(x, \xi, \lambda)\right| \leq & \text { const } \sum_{|\alpha| \geq 1} \sum_{k \neq j}(1+|\xi|)^{l_{i}+m_{k}-|\alpha|+l_{k}+m_{j}} \\
& \times\left(|\xi|^{r_{k}}+|\lambda|\right)^{-1}\left(|\xi|^{r_{j}}+|\lambda|\right)^{-1} \\
& + \text { const } \sum_{|\alpha| \geq 1}(1+|\xi|)^{l_{i}+m_{j}-|\alpha|}\left(|\xi|^{r_{j}}+|\lambda|\right)^{-1} \\
\leq & \operatorname{const}(1+|\xi|)^{l_{i}+m_{j}-1}\left(|\xi|^{r_{j}}+|\lambda|\right)^{-1} .
\end{aligned}
$$


Differentiating (5.24) consecutively with respect to $x$, we obtain the estimate

$$
\left|D_{x}^{\beta} T_{i j}(x, \xi, \lambda)\right| \leq \operatorname{const}(1+|\xi|)^{l_{i}+m_{j}-1}\left(|\xi|^{r_{j}}+|\lambda|\right)^{-1} .
$$

The last inequality permits us to estimate the left-hand side of (5.23) by

$$
\text { const }(1+|\xi|)^{l_{i}+m_{j}-1}\left(|\xi|^{r_{i}}+|\lambda|\right)^{-\frac{l_{i}}{r_{i}} \vartheta\left(r_{i}\right)}\left(|\xi|^{r_{j}}+|\lambda|\right)^{-\frac{m_{j}}{r_{j}} \vartheta\left(r_{j}\right)}
$$

If $r_{i}>0, r_{j}>0$ the expression (5.27) is not greater than

$$
\begin{aligned}
\operatorname{const}(1+|\xi|)^{l_{i}+m_{j}-1}\left(|\xi|+|\lambda|^{1 / r_{i}}\right)^{-l_{i}}\left(|\xi|+|\lambda|^{1 / r_{j}}\right)^{-m_{j}} & \leq \operatorname{const}\left(|\xi|+|\lambda|^{\varepsilon}\right)^{-1} \\
& \leq \operatorname{const}|\lambda|^{-\varepsilon}
\end{aligned}
$$

with $\varepsilon=\min \left(1 / r_{i}, 1 / r_{j}\right)$. The same estimate holds when either $r_{j}>0, r_{i}=0$, and $\varepsilon=1 / r_{j}$, or $r_{i}>0, r_{j}=0, \varepsilon=1 / r_{i}$. In the case $r_{i}=r_{j}=0$ we have

$$
\left|D^{\beta} T_{i j}(x, \xi, \alpha)\right|<\text { const }|\lambda|^{-1} \text {. }
$$

Thus, we have proved (5.23) with $\varepsilon_{i j \beta}(\lambda)=\varepsilon_{i j \beta} \lambda^{-\varepsilon}$, where $\varepsilon>0$.

(iii) We give only a brief sketch of the proof. According to the commutation formula for pseudodifferential operators,

$$
G(x, D, \lambda)(A(x, D)-\lambda I)-I=T^{(N)}(x, D, \lambda)+R^{(N)},
$$

for arbitrary $N=1,2, \ldots$, where $T^{(N)}$ is a pseudodifferential operator with the symbol

$$
\sum_{|\alpha|=1}^{N-1} \frac{1}{\alpha !} \partial_{\xi}^{\alpha} G(x, \xi, \lambda) D_{x}^{\alpha} A(x, \xi)
$$

and $R^{(N)}$ is a pseudodifferential operator with the symbol

$$
(2 \pi)^{-n} \sum_{|\alpha|=N} \int_{0}^{1} \iint_{\mathbb{R}^{2 n}} \exp (i(x-y) \cdot(\eta-\xi)) \partial_{\xi}^{\alpha} G(x, \xi+t(\eta-\xi))\left(D_{x}^{\alpha} A\right)(y, \xi) d y d \eta d t
$$

The following estimates (cf. (5.15), (5.16)) are important for the proof:

(5.30) $\left|\partial_{\xi}^{\alpha} D_{x}^{\beta} G_{i j}(x, \xi, \lambda)\right| \leq C_{i j \alpha \beta}(1+|\xi|)^{l_{i}+m_{j}-|\alpha|}\left(|\xi|^{r_{i}}+|\lambda|\right)^{-1}\left(|\xi|^{r_{j}}+|\lambda|\right)^{-1} \quad(i \neq j)$,

$$
\left|\partial_{\xi}^{\alpha} D_{x}^{\beta} G_{i i}(x, \xi, \lambda)\right| \leq C_{i i \alpha \beta}(1+|\xi|)^{-|\alpha|}\left(|\xi|^{r_{i}}+|\lambda|\right)^{-1}
$$

Using these estimates and repeating the argument used in the proof of (ii) we show that (cf. (5.26))

$$
\left(|\xi|^{r_{i}}+|\lambda|\right)^{\frac{m_{i}}{r_{i}}} D_{x}^{\beta} T_{i j}^{(N)}(x, \xi, \lambda)\left(|\xi|^{r_{j}}+|\lambda|\right)^{-\frac{m_{j}}{r_{j}}} \leq C_{\beta i j}(\lambda)
$$

and the constant on the right - hand side tends to zero as $|\lambda| \rightarrow \infty$. From the last estimate it follows that the operator (5.11) with $G(A-\lambda I)-I$ replaced by $T^{(N)}$ has norm less than 1 for large $|\lambda|$. 
As for the remainder term $R^{(N)}$, the expression in the integrand (5.29) has a compact support with respect to $y$ and decreases as const $(1+|\xi+t(y-\xi)|)^{-N}$. Standard estimates of the calculus of pseudodifferential operators permit us to estimate the norm of (5.11) with $G(A-\lambda I)-I$ replaced by $R^{(N)}$.

To prove (5.30), (5.31) we use (5.17). For $|\alpha|=1, \beta=0$ we obtain

$$
\partial_{\xi}^{\alpha} G_{i j}=-\left(\partial_{\xi}^{\alpha} P P^{-2}\right) \operatorname{det}\left((A-\lambda I)^{j i}\right)+P^{-1} \partial_{\xi}^{\alpha} \operatorname{det}\left((A-\lambda I)^{j i}\right) .
$$

From this relation $(5.30)$, (5.31) follows for $|\alpha|=1, \beta=0$. Differentiating successively the last relation, we obtain the desired estimates.

\subsection{Proof of Proposition 5.5}

We fix $x^{0} \in \mathbb{R}^{n}, \kappa=1, \ldots, q$, and choose the numbers $r$ and $\mu_{j}(\kappa)$ according to (4.13), (4.14).

We replace $\lambda$ in $(5.7)$ by $\rho^{r} \lambda$ and $u(x)$ by $u_{\rho}(x)=\left(\left(u_{1 \rho}(x), \ldots, u_{q \rho}(x)\right)\right.$, where

$$
u_{j \rho}(x)=\rho^{\frac{n}{2}-\mu_{j}(\kappa)} u_{j}\left(\rho\left(x-x^{0}\right)\right) \quad(j=1, \ldots, q) .
$$

As it was shown in the proof of Lemma 4.2, the limit of the left - hand side of (5.7) as $\rho \rightarrow+\infty$ is equal to

$$
\left(\sum_{j=1}^{\kappa}\left\||D|^{m_{j}} u_{j}\right\|^{2}+\sum_{j=\kappa+1}^{q}|\lambda|^{\frac{2 m_{j}}{r_{j}}}\left\|u_{j}\right\|^{2}\right)^{\frac{1}{2}} .
$$

Now we shall calculate the limit of the right-hand side. Without loss of generality we suppose that $u_{j}(x) \in \mathcal{D}, j=1, \ldots, q$. Set

$$
f_{j \rho}(x)=\sum_{k=1}^{q} \rho^{\frac{n}{2}-\mu_{k}(\kappa)} A_{j k}(x, D) u_{k}\left(\rho\left(x-x^{0}\right)\right)-\lambda \rho^{\frac{n}{2}-\mu_{j}(\kappa)+r} u_{j}\left(\rho\left(x-x^{0}\right)\right) .
$$

The right - hand side of (5.7) can written as the square root of

$$
\begin{aligned}
& \sum_{j} \int\left(|\xi|^{2 r_{j}}+\rho^{2 r}|\lambda|^{2}\right)^{-\frac{l_{j}}{r_{j}}}\left|\widehat{f}_{j \rho}(\xi)\right|^{2} d \xi \\
= & \sum_{j} \int\left(\rho^{2 r_{j}}|\xi|^{2 r_{j}}+\rho^{2 r}|\lambda|^{2}\right)^{-\frac{l_{j}}{r_{j}}} \rho^{n}\left|\widehat{f}_{j \rho}(\rho \xi)\right|^{2} d \xi \\
= & \sum J_{\rho j}^{2} .
\end{aligned}
$$

Consider the term corresponding to $j \leq \kappa$. We have

$$
J_{\rho j}^{2}=\int\left(|\xi|^{2 r_{j}}+\rho^{2\left(r-r_{j}\right)}|\lambda|^{2}\right)^{-\frac{l_{j}}{r_{j}}}\left|\rho^{n / 2-l_{j}} \widehat{f}_{j \rho}(\rho \xi)\right|^{2} d \xi .
$$

Now we show that

$$
\rho^{n / 2-l_{j}}\left|\widehat{f}_{j \rho}(\rho \xi)\right| \longrightarrow\left|\sum_{k=1}^{\kappa} A_{j k}^{0}\left(x^{0}, \xi\right) \widehat{u}_{k}(\xi)\right| \quad(\rho \rightarrow+\infty) .
$$


According to the definition of the Fourier transform,

$$
\begin{aligned}
& \rho^{n / 2-l_{j}} \widehat{f}_{j \rho}(\rho \xi) \\
= & (2 \pi)^{-n / 2} \int \exp (-i \rho \xi \cdot x) \\
& \times\left(\sum_{k} \rho^{n-l_{j}-\mu_{k}(\kappa)} A_{j k}(x, D) u_{k}\left(\rho\left(x-x^{0}\right)\right)-\lambda \rho^{n-\mu_{j}(\kappa)+r-l_{j}} u_{j}\left(\rho\left(x-x^{0}\right)\right)\right) d x .
\end{aligned}
$$

If we set $x=x^{0}+\rho^{-1} y$ in the integrand we obtain

$$
\begin{aligned}
& \rho^{n / 2-l_{j}} \widehat{f}_{j \rho}(\rho \xi) \\
= & (2 \pi)^{-n / 2} \exp \left(-i \rho \xi \cdot x^{0}\right) \int \exp (-i \xi \cdot y) \\
& \times\left(\sum_{k} A_{j k}\left(x^{0}+\rho^{-1} y, \rho D_{y}\right) \rho^{-l_{j}-\mu_{k}(\kappa)} u_{k}(y)-\lambda \rho^{-\mu_{j}(\kappa)+r} u_{j}(y)\right) d y .
\end{aligned}
$$

Passing to the limit in the integral as $\rho \rightarrow+\infty$ we obtain (5.34). As a result we prove that expression (5.33) tends to

$$
\sum_{j=1}^{\kappa}\left\||D|^{-l_{j}} \sum_{k=1}^{\kappa} A_{j k}^{0}(D) u_{k}\right\|^{2}+\sum_{j=\kappa+1}^{q}|\lambda|^{\frac{2 m_{j}}{r_{j}}}\left\|u_{j}\right\|^{2} .
$$

Setting $u_{\kappa+1} \equiv \cdots \equiv u_{q} \equiv 0$ we obtain inequality (4.10). Inequality (4.11) can be proved in the same way.

\subsection{Ellipticity with parameter for systems on a compact manifold without boundary}

Let $M$ be a smooth compact $n$-dimensional manifold without boundary. It is wellknown that the Sobolev spaces $H^{s}(M)$ can be defined for arbitrary real $s$. Thus, we can define the spaces $U(M)=\prod H^{m_{j}}(M)$ and $F(M)=\prod H^{-l_{j}}(M)$. In these spaces the analogues of the norms $(4.3),(4.4),(4.6)$ can be defined, and the corresponding spaces will be denoted by $U(M, \lambda)$ and $F(M, \lambda)$.

The matrix differential operator $A(x, D)$ defined for $x \in M$ will be considered as a continuous operator

$$
U(M, \lambda) \longrightarrow F(M, \lambda) \quad(u \mapsto A(x, D) u)
$$

Since Definition 5.1 concerns the principal symbol, one can trivially reformulate it for operators defined on $M$. The standard localization technique used in the construction of parametrices of elliptic operators on manifolds permits us to prove the exact analogue of Theorem 5.3.

Theorem 5.6. For the matrix operator $A(x, D)$ with $C^{\infty}$ coefficients defined on a smooth compact manifold $M$ the following conditions are equivalent. 
(A) The principal symbol $A^{0}(x, \xi)-\lambda I$ satisfies the conditions of Definition 5.1 for each $x \in M$.

(B) There exists a ray (1.1) and $\rho_{0}>0$ such that for $\lambda \in \mathcal{L}$ with $|\lambda| \geq \rho_{0}$ and for arbitrary $f \in T(M, \lambda)$ the equation

$$
A(x, D) u-\lambda u(x)=f(x)
$$

has a unique solution $u \in U(\lambda)$, and the estimate

$$
\|u, U(M, \lambda)\| \leq K\|f, F(M, \lambda)\|
$$

holds with a constant $K$ independent of $\lambda$.

$\left(\mathrm{B}_{0}\right)$ There exists a ray $(1.1)$ and a number $\rho_{0}>0$ such that for $u_{j} \in C^{\infty}(M), j=$ $1, \ldots, q$, the a priori estimate

$$
\|u, U(M, \lambda)\| \leq K\|A(x, D) u-\lambda u, F(M, \lambda)\| \quad\left(\lambda \in \mathcal{L},|\lambda| \geq \rho_{0}\right)
$$

holds with $K$ independent of $\lambda$.

\section{Acknowledgements}

The authors would like to express their deep gratitude to M. S. AGRANOVICH for fruitful discussions and very useful remarks concerning the exposition.

The support of the Deutsche Forschungsgemeinschaft is gratefully acknowledged. The paper was written during the stay of the third author in Regensburg under the grant of the Deutsche Forschungsgemeinschaft.

Added in proof. In [K3] some $2 \times 2$ block systems which are elliptic with parameter are considered on a manifold with boundary. The boundary conditions are assumed to be homogeneous and of triangular form. For this problem the existence of the resolvent is announced.

\section{References}

[A] Agranovich, M. S.: Elliptic Operators on Closed Manifolds, Encyclopaedia Math. Sci. 63, Springer - Verlag, 1994

[Agm] Agmon, S.: On the Eigenfunctions and on the Eigenvalues of General Elliptic Boundary Value Problems, Comm. Pure Appl. Math. 15 (1962), 119-147

[AV] Agranovich, M. S., and Vishik, M. I.: Elliptic Problems with Parameter and General Type Parabolic Problems, Uspekhi. Mat. Nauk 19 (1964), No. 3, 53-161; English transl. in Russian Math. Surveys 19 (1964), 53-157

[GG] Grubb, G., and Geymonat, G.: The Essential Spectrum of Elliptic Systems of Mixed Order, Math. Ann. 227 (1977), $247-276$

[GV] Gindikin, S. G., and Volevich, L. R.: The Method of Newton's Polyhedron in the Theory of Partial Differential Equations, Math. Appl. (Soviet Ser.) 86, Kluwer Academic, Dordrecht, 1992

[G] GrubB, G.: Functional Calculus of Pseudo-Differential Boundary Problems, Birkhäuser, Boston-Basel-Stuttgart, 1986 
[H] Hardy, G. H., Littlewood, J. E., And Pólya, G.: Inequalities, Second edition, Cambridge University Press, Cambridge, 1952

[K1] Kozhevnikov, A.: Spectral Problems for Pseudo-Differential Systems Elliptic in the Douglis - Nirenberg Sense, and Their Applications, Mat. USSR Sb. 21 (1973), 63-90

[K2] Kozhevnikov, A.: Asymptotics of the Spectrum of Douglis - Nirenberg Elliptic Operators on a Closed Manifold, Math. Nachr. 182 (1996), 261-293

[K3] Kozhevnikov, A.: Parameter-Ellipticity for Mixed-Order Elliptic Boundary Problems, C. R. Acad. Sci. Paris Sér. I Math. 324 (1997), 1361-1366

[Sh] Shubin, M. A.: Pseudo-Differential Operators and Spectral Theory, Springer-Verlag, Berlin, 1987

[V1] Volevich, L. R.: On General Systems of Differential Equations, Dokl. Akad. Nauk SSSR 132 (1960), No 1, 20-23; English transl. in Soviet Math. Dokl. 1 (1960), 458-461

[V2] Volevich, L. R.: On a Linear Programming Problem Arising in Partial Differential Equations, Uspekhi Mat. Nauk 18 (1963), No. 3, 155-162

Universität Regensburg

NWF I - Mathematik

D-93040 Regensburg

Germany

e-mail:

robert.denk@mathematik.uni-regensburg.de

reinhard.mennicken@mathematik.uni-regensburg.de
Keldysh Institute

of Applied Mathematics

Russian Acad. Sci.

Miusskaya sqr. 4

125047 Moscow

Russia

e-mail:

volevich@spp.keldysh.ru 